\title{
Ergodicity and Energy Distributions for Some Boundary Driven Integrable Hamiltonian Chains
}

\author{
Peter Balint ${ }^{1}$, Kevin K. Lin ${ }^{2}$, and Lai-Sang Young ${ }^{3}$
}

\begin{abstract}
We consider systems of moving particles in 1-dimensional space interacting through energy storage sites. The ends of the systems are coupled to heat baths, and resulting steady states are studied. When the two heat baths are equal, an explicit formula for the (unique) equilibrium distribution is given. The bulk of the paper concerns nonequilibrium steady states, i.e., when the chain is coupled to two unequal heat baths. Rigorous results including ergodicity are proved. Numerical studies are carried out for two types of bath distributions. For chains driven by exponential baths, our main finding is that the system does not approach local thermodynamic equilibrium as system size tends to infinity. For bath distributions that are sharply peaked Gaussians, in spite of the near-integrable dynamics, transport properties are found to be more normal than expected.
\end{abstract}

\section{Introduction}

This paper concerns a simple model of energy transport along a chain of Hamiltonian systems with conservative, nearest-neighbor coupling. The ends of the chain are in contact with unequal heat baths, and of interest to us are properties of the resulting nonequilibrium steady state. In this paper, we address rigorously questions of invariant densities and ergodicity. This is followed by a numerical investigation of energy profiles along the chain and marginal distributions at specific locations as the length of the chain tends to infinity.

The setup above is used in heat conduction studies, as are the questions raised [3, 11, 21]. We propose to look at these questions in a broader theoretical framework, as transport of conserved quantities by dynamical processes in general and particle systems in particular. As an example of this extended viewpoint, we will sometimes consider energy sources that are not necessarily Gibbsian in distribution, even though we will continue to call them "heat baths".

Except for system-bath interactions, the class of models considered here obey

\footnotetext{
${ }^{1}$ Institute of Mathematics, Budapest University of Technology and Economics, H-1111 Egry József u. 1 Budapest, Hungary. Email: pet@math.bme.hu. Research partially supported by OTKA (Hungarian National Research Fund) grants: F 60206 and K 71693; and by the Bolyai scholarship of the Hungarian Academy of Sciences.

${ }^{2}$ Department of Mathematics, University of Arizona, Tucson, AZ 85721, USA. Email: klin@math.arizona.edu

${ }^{3}$ Courant Institute of Mathematical Sciences, New York University, New York, NY 10012, USA. Email: lsy@cims.nyu.edu. This research is supported by a grant from the NSF
} 
purely deterministic (as opposed to stochastic) microscopic rules. ${ }^{4}$ Transport properties naturally depend on these dynamical rules. It is generally thought - though far from proved - that "chaotic" local dynamics lead to diffusion-like behavior on the macroscopic level, while "integrable" dynamics, which lie at the opposite end of the spectrum, operate differently. An early example that contributes to this understanding is a chain of coupled harmonic oscillators [28]; see [21] for other examples and more detailed discussion. The class of models considered in this paper is closer to the integrable end.

Description of model. The constituent subsystems in our chains are as simple as can be: each consists of a point mass moving freely back and forth along a unit length interval. We order these intervals linearly. Energy is stored at the sites where adjacent intervals meet; when a particle reaches a site, it exchanges energy with the site, then turns around and moves away at a speed equal to the square root of its new (kinetic) energy. The left and right ends of the chain are coupled to heat baths, which function like other sites except that the energies they emit are random; we assume they are i.i.d. with respect to certain prescribed distributions. This is a complete description of the class of models studied in this paper.

If we replace the two baths by two regular sites, then the resulting closed chain is a very simple, totally non-chaotic dynamical system: it has many independent conserved quantities (besides the total energy) and is highly non-ergodic. For this reason, we will sometimes refer to it (loosely) as an "integrable Hamiltonian chain". Any ergodic property of the open chain must come solely from the randomness of the baths, and this randomness must find a way to penetrate the entire chain, which can be arbitrarily long.

Preview of results. This paper has two separate parts: a rigorous part, and a numerical part.

Our main rigorous result is ergodicity; under some conditions which require that the bath distributions be sufficiently spread out, we prove that there is at most one invariant probability measure, and that this measure has a density. In particular, we prove that our system admits no singular invariant probability measures. We also give the explicit form of the invariant density when the system is in equilibrium, i.e., when the two bath distributions are equal.

Numerical studies of nonequilibrium steady states were carried out for two types of bath distributions: exponential distributions and sharply peaked Gaussians. When in equilibrium, exponential bath distributions give rise to Gibbs distributions for the moving particles in the chain. A finding that was a little surprising to us is that when forced out of equilibrium, our chain produces local marginal densities that are mixtures of Gibbs distributions (which are therefore not Gibbs). This is discussed in Sect. 6.2. Sharply peaked Gaussian bath distributions were chosen to accentuate the

\footnotetext{
${ }^{4}$ There is a small exception to this statement: a stochastic rule is used to "break the tie" in the unlikely event of same-site double collisions; see the end of Sect. 1.1.
} 
near-integrability of the dynamics. Here we discover that injected energies move about in a hybrid ballistic-diffusive motion, resembling a random walk with "momentum", meaning a tendency to continue in the same direction; see Sect. 7.2.

Related works. With regard to ergodicity type results for boundary driven Hamiltonian chains, the only previous works we are aware of are for anharmonic chains $[15$, $12,27]$ and for certain particle models in which rotating disks are used as energy storage devices [13]. The latter was preceded by [20,25], which contain numerical studies of similar models, and by [16], which contains a simplification of the model in [20] and a partial analysis. A similar class of models, with paddles in the place of rotating disks, has also been studied [22]. These models have to some degree motivated the idea of energies at "sites" in the present paper. Related works in systems defined by dynamical local rules include $[4,5,6,7,17]$. For stochastic models, the collection of results is much larger, and we mention only some that are closer to this paper in spirit: $[1,2,8,9,10,16,18,19,23,24,26,29]$.

\section{Model Description}

\subsection{Particle systems}

We consider a model with $N$ sites, denoted $\{1,2, \cdots, N\}$, connected to two baths which we think of as located at sites 0 and $N+1$. The physical space of the system is the line segment $[0, N+1]$. In this system, there are $2 N+1$ particles, $N$ of which are "stationary" and the rest are in perpetual motion. The $i$ th stationary particle is confined to site $i$, while the $i$ th moving particle, $i=0,1, \cdots, N$, moves back and forth along the segment $[i, i+1]$. Each particle carries an energy. The energy of the $i$ th stationary particle is denoted by $\xi_{i}$, while the $i$ th moving particle carries energy $\eta_{i}$ and moves with speed $\sqrt{\eta_{i}}$. The phase variables of this system are therefore

$$
X=\left(\left(x_{0}, \sigma_{0}, \eta_{0}\right), \xi_{1},\left(x_{1}, \sigma_{1}, \eta_{1}\right), \xi_{2},(\cdots), \cdots \xi_{N},\left(x_{N}, \sigma_{N}, \eta_{N}\right)\right) \text {. }
$$

Here we let $x_{i}(t) \in[0,1]$ denote the location at time $t$ of the $i$ th moving particle and $\sigma_{i}(t)= \pm$ its direction of travel, identifying the segments $[i, i+1]$ with $[0,1]$. The variables $\eta_{i}, \xi_{i} \in(0, \infty)$ are energies as defined earlier. Because the moving particles are trapped in gaps between integer sites, we will also refer to the $\eta_{i}$ as "gap energies."

The rules for updating this continuous-time process are as follows. When a moving particle reaches the boundary of the segment to which it is confined, a collision occurs, and the post-collision dynamics are defined as follows: Suppose for definiteness that at time $t_{0}$,

$$
x_{i}\left(t_{0}^{-}\right)=0, \quad \sigma_{i}\left(t_{0}^{-}\right)=-; \quad i \neq 0,
$$

i.e., at time $t_{0}$, the $i$ th particle collides with site $i$, which we assume is not the left bath. We assume further that $x_{i-1}\left(t_{0}^{-}\right) \neq 1$, i.e., it is not a double collision at site $i$. 
Then an exchange of energy between the particle at site $i$ and the $i$ th moving particle takes place, and the latter reverses its direction, i.e.,

$$
\xi_{i}\left(t_{0}^{+}\right)=\eta_{i}\left(t_{0}^{-}\right), \quad \eta_{i}\left(t_{0}^{+}\right)=\xi_{i}\left(t_{0}^{-}\right), \quad x_{i}\left(t_{0}^{+}\right)=0, \quad \text { and } \quad \sigma_{i}\left(t_{0}^{+}\right)=+.
$$

An analogous rule holds when $x_{i}\left(t_{0}^{-}\right)=1$ and $\sigma_{i}^{-}\left(t_{0}\right)=+$ provided that $i \neq N$.

When a particle reaches a bath, it bounces back with a random energy. More precisely, we think of the left and right baths as having energy distributions with densities $L(\xi)$ and $R(\xi)$ respectively. Each time the 0th moving particle reaches the left bath, its energy is replaced by one drawn randomly and independently from the distribution $L(\xi)$; an analogous statement holds when the $N$ th moving particle reaches the right bath. Emissions from the left and right baths are independent.

We say a multiple collision occurs at time $t_{0}$ if at that time, two or more collisions occur simultaneously. These collisions may involve the same site, or different sites, or some combination of sites and baths. The post-collision rule above leaves no ambiguity as to how to continue the dynamics after a multiple collision except when there is a "same-site double collision", i.e., when two particles arrive, from the left and the right, to one of the sites at exactly the same time. There is no natural way to continue the dynamics after such a collision. We fix (arbitrarily) the following rule: equal probability is assigned to the two scenarios corresponding to one of the particles arriving slightly ahead of the other; if collisions of this type occur simultaneously at multiple sites, then the probabilities are assigned independently at each site.

\subsection{Markov jump processes with very degenerate transitions}

The process described in Sect. 1.1 is a Markov jump process. One may represent such a process as a unit speed flow built over the "jump section". Let $\mathcal{M}^{+}$denote the set of configurations $X$ immediately following a collision. For each $X \in \mathcal{M}^{+}$, let $r(X)$ denote the time to the next collision, and let

$$
\mathcal{M}:=\left\{(X, s): X \in \mathcal{M}^{+} \text {and } s \in[0, r(X)]\right\}
$$

Transition probabilities $P^{t}$ for the Markov process on $\mathcal{M}$ are defined as follows:

- for $t<r(X), P_{(X, 0)}^{t}=\delta_{(X, t)}$, the point mass at $(X, t)$;

- for $t=r(X)$, a jump occurs, and $P_{(X, 0)}^{t}$ is the probability measure supported on $\mathcal{M}^{+} \times\{0\}$ given by the rules in the last subsection.

Geometrically, each point in $\mathcal{M}^{+} \times\{0\}$ moves "up" in the second coordinate at unit speed until it reaches the pre-jump section $\{(X, s): s=r(X)\}$, at which time its image is identified, instantaneously, with a distribution on $\mathcal{M}^{+} \times\{0\}$. This distribution is a point mass a vast majority of the time. It has a density in one direction if the collision involves exactly one of the baths, in two directions if both baths are 
involved, and it is supported on a finite set of points following a same-site double collision. Transition probabilities at other points in $\mathcal{M}$ are defined in the obvious way.

The continuous-time Markov process above induces a discrete-time Markov chain on $\mathcal{M}^{+}$defined as follows: For $X \in \mathcal{M}^{+}$, the (time-1) transition probability starting from $X$, denoted $p_{X}$, is given by $p_{X}(A)=P_{(X, 0)}^{r(X)}(A \times\{0\})$ for all $A \subset \mathcal{M}^{+}$.

A Borel probability measure $\mu$ on $\mathcal{M}^{+}$is said to be invariant under the discretetime Markov chain $p^{n}$ if for all Borel sets $A \subset \mathcal{M}^{+}$,

$$
\mu(A)=\int p_{X}(A) d \mu(X)
$$

Invariant measures for the continuous-time process $P^{t}$ are defined similarly. The invariant measures of these two processes are related via the following elementary lemma the proof of which we omit. Let $\lambda_{k}$ denote Lebesgue measure on $\mathbb{R}^{k}$.

Lemma 1.1 (a) For every Borel probability measure $\mu$ on $\mathcal{M}^{+}$invariant under $p^{n}$, $\hat{\mu}:=\left.\left(\mu \times \lambda_{1}\right)\right|_{\mathcal{M}}$ is invariant under $P^{t} ; \hat{\mu}(\mathcal{M})<\infty$ if and only if $\int r d \mu<\infty$.

(b) Conversely, each $P^{t}$-invariant probability measure $\hat{\mu}$ gives rise to a $p^{n}$-invariant measure $\mu$ defined by

$$
\mu(A)=\lim _{\varepsilon \rightarrow 0} \frac{1}{\varepsilon} \hat{\mu}(\{(X, s): X \in A, s \in[0, \varepsilon]\} .
$$

\subsection{The roaming particles viewpoint}

In Sect. 1.1, we have described our model as consisting of $N+1$ moving particles each confined to a unit interval, exchanging energy with the particles confined to sites when they meet. The following entirely equivalent way of viewing this model is sometimes convenient and is used in many of the arguments:

Think of each particle as carrying an energy which remains unchanged throughout, i.e., there are no energy exchanges. Instead, the particles exchange positions when they meet. More precisely, a particle may be moving or at rest; when at rest, it is at one of the sites, and is the sole occupant of that site. When a moving particle reaches site $i$, it exchanges positions with the particle sitting at site $i$, and waits there until it is relieved by the next particle to reach this site. Each particle is permitted to roam the length of the chain until it exits at one of the two ends.

Formally, to define an equivalence with the model described in Sect. 1.1, one should view all particles carrying the same energy as indistinguishable. Notice that this does not prevent one from following, with no ambiguity, the trajectory of a particle from the moment it enters the chain to when it leaves - except in the case of same-site double collisions involving 3 particles with the same energy. In such a collision, one 
cannot deduce from the subsequent dynamics which one of the approaching particles is assumed to have arrived first.

In this roaming particles description of the model, there is also the induced discrete-time process, which is defined in the obvious way.

\section{PART I. RIGOROUS RESULTS}

\section{Statement of results}

Our results pertain to invariant measures of the processes defined in Sect.1. When the bath distributions $L(\xi)$ and $R(\xi)$ are equal, we regard the system as being in equilibrium, and view its invariant measures as equilibrium distributions. When $L(\xi) \neq R(\xi)$, we view the system as forced out of equilibrium, and its invariant measures as nonequilibrium steady states.

Our first result applies to situations both in and out of equilibrium. As noted in the introduction, the closed chain corresponding to our model is integrable and highly nonergodic, so that ergodicity of the open system, if true, is brought about solely by the randomness from the baths. Intuitively, the more spread out the injected energies, the more likely ergodicity will be achieved.

Theorem 1 Assume $L(\xi), R(\xi)>0$ for all $\xi \in(0, \infty)$. Then the following hold for all $N$-chains for both the continuous-time process $P^{t}$ and its associated discrete-time process $p^{n}$ :

(a) There is at most one invariant probability measure (which is therefore ergodic).

(b) This measure has a density with respect to Lebesgue measure.

The conditions above can be weakened as follows: There is a function $\phi(N)=O\left(\frac{1}{N^{2}}\right)$ such that (a) and (b) hold for the $N$-chain if $L(\xi)$ and $R(\xi)$ are strictly positive on open sets $I_{L}$ and $I_{R}$, and there exist $\xi \in I_{L} \cup I_{R}$ and $\xi^{\prime} \in I_{L} \cap I_{R}$ such that $\xi=\phi(N) \xi^{\prime}$.

An example of $\phi(N)$ that is sufficient is $\phi(N)=\frac{1}{(18 N)^{2}}$. The form of our assumption involving $\phi(N)$ is quite possibly an artifact of our proof, but it is essential for $L(\xi)$ and $R(\xi)$ to have some spread. Our results imply, in particular, that the processes we consider have no singular invariant probability measures.

We do not prove the existence of invariant measures in this paper. For existence, the two issues are: (1) tightness, which requires that one controls the dynamics of super-fast and super-slow particles. (2) Discontinuities of transition probabilities $p_{X}$ as a function of $X$ due to same-site double collisions. Techniques for treating (1) in nonequilibrium situations are generally lacking, and (2) is likely to involve very technical arguments. We have elected to leave these problems for future work. 
Our next result gives an explicit formula for the equilibrium distribution in terms of bath distributions. In general, no such explicit expressions exist for nonequilibrium steady states, and our models appear to be no exception.

Theorem 2 Let $L(\xi)=R(\xi)=\rho(\xi)$ be a probability density on $(0, \infty)$ satisfying ( $i)$ $\int \frac{1}{\sqrt{\xi}} \rho(\xi) d \xi<\infty$ and (ii) $\rho(\xi)=O\left(\xi^{-\kappa}\right)$ for some $\kappa>3 / 2$ as $\xi \rightarrow \infty$. Then the following measure is invariant for the (continuous-time) process defined in Sect. 1.1:

$$
\left(d \xi_{1} \cdots d \xi_{N} \cdot \prod_{i=1}^{N} \rho\left(\xi_{i}\right)\right) \times\left(d \eta_{0} \cdots d \eta_{N} \cdot \prod_{i=0}^{N} \frac{1}{\sqrt{\eta_{i}}} \rho\left(\eta_{i}\right)\right) \times \lambda_{N+1} \times \omega_{N+1}
$$

where $\lambda_{N+1}=$ Lebesgue measure on $[0,1]^{N+1}$ and $\omega_{N+1}$ assigns equal weight to the configurations in $\left\{\left(\sigma_{1}, \cdots, \sigma_{N+1}\right)\right\}$.

The following corollary suggests some examples to which our results apply:

Corollary 1. Any finite chain with bath injections $L(\xi)=R(\xi)$ given by either

(a) an exponential distribution or

(b) a Gaussian truncated at 0 and normalized

has a unique (hence ergodic) equilibrium distribution, and it is given by the expression in Theorem 2.

\section{Density and Ergodicity: Outline of Proof}

For definiteness, we will work with the discrete-time Markov chain on $\mathcal{M}^{+}$. By Lemma 1.1, the assertions in Theorem 1 for $p^{n}$ imply the corresponding assertions for $P^{t}$.

Proving uniqueness of invariant measure or ergodicity requires, roughly speaking, that we be able to steer a trajectory from one location of the phase space to another. If the transition probabilities have densities on open sets, then one needs only to do so in an approximate way. Our model, unfortunately, has highly degenerate transition probabilities. One must, therefore, tackle hand in hand the problems of (i) acquisition of densities for $p_{X}^{n}$ and (ii) steering of trajectories.

The purpose of this section is to outline how we plan to do this. Sect. 3.1 introduces definitions and ideas that will be used. In Sect. 3.2, we formulate three propositions to which the proof of Theorem 1 will be reduced.

\subsection{Basic ingredients of the proof}

\section{(A) Acquiring densities}

Given a finite Borel measure $\nu$ on $\mathcal{M}^{+}$, we let $\nu=\nu_{\perp}+\nu_{a c}$ denote the decomposition of $\nu$ into a singular and an absolutely continuous part with respect to Lebesgue measure, and say $\nu$ has an absolutely continuous component when $\nu_{a c} \neq 0$. We 
say $X \in \mathcal{M}^{+}$eventually acquires a density if for some $n>0, p_{X}^{n}$ has an absolutely continuous component, i.e. $\left(p_{X}^{n}\right)_{a c} \neq 0$.

Lemma 3.1 If every $X \in \mathcal{M}^{+}$acquires a density eventually, then every invariant probability measure of $p^{n}$ has a density.

Proof: For any measure $\nu$, if $\nu$ is absolutely continuous with respect to Lebesgue, then so is $\nu_{n}:=\int p_{X}^{n} d \nu(X)$. This is because under the dynamics, Lebesgue measure is carried to a measure equivalent to Lebesgue, followed, at certain steps, by a diffusion in one or two directions corresponding to bath injections. Thus $\left(\nu_{n}\right)_{a c}\left(\mathcal{M}^{+}\right) \geq \nu_{a c}\left(\mathcal{M}^{+}\right)$ for all $n \geq 1$. The hypothesis of this lemma implies that this inequality is strict for some $n$ unless $\nu_{\perp}=0$. Now let $\mu$ be an invariant probability measure for $p^{n}$. Since $\mu_{n}=\mu$, it follows that $\mu_{\perp}=0$.

Recall that $I_{L}=\{L>0\}$ and $I_{R}=\{R>0\}$. We call a finite or infinite sequence of points $X_{0}, X_{1}, \ldots$ in $\mathcal{M}^{+}$a sample path if such a sequence can, in principle, occur. In particular, if $X_{n-1}$ is followed by a collision with the right bath, then the $\xi_{N^{-}}$ coordinate of $X_{n}$ must lie in $I_{R}$, and similarly with the left bath.

Let $\underline{X}=\left(X_{0}, X_{1}, \ldots, X_{n}\right)$ be a sample path obtained by injecting into the system the energy sequence $\underline{\varepsilon}=\left(\varepsilon_{1}, \cdots, \varepsilon_{m}\right)$ in the order shown. (One does not specify whether the injection is from the left or the right; it is forced by the sequence.) We write $\Phi_{X_{0}}(\underline{\varepsilon})=X_{n}$. Suppose $\underline{X}$ has no multiple collisions. It is easy to see that there is a small neighborhood $\mathcal{E}$ of $\underline{\varepsilon}$ in $\mathbb{R}^{m}$ such that for all $\underline{\varepsilon}^{\prime} \in \mathcal{E}$, (i) $\underline{\varepsilon}^{\prime}$ is a feasible sequence of injections, i.e., if the sequence dictates that $\varepsilon_{j}^{\prime}$ be injected from the left (right) bath, then $\varepsilon_{j}^{\prime} \in I_{L}$ (resp. $I_{R}$ ), and (ii) the sample path $\underline{X}^{\prime}=\left(X_{0}^{\prime}, X_{1}^{\prime}, \ldots, X_{n}^{\prime}\right)$ produced by injecting $\underline{\varepsilon}^{\prime}$ has the exact same sequence of collisions as $\underline{X}$. In particular, it also has no multiple collisions. We may therefore extend $\Phi=\Phi_{X_{0}}$ to a mapping from $\mathcal{E}$ to $\mathcal{M}^{+}$with $\Phi\left(\underline{\varepsilon}^{\prime}\right)=X_{n}^{\prime}$. As such, $\Phi$ is clearly continuous.

Lemma $3.2 \Phi: \mathcal{E} \rightarrow \mathcal{M}^{+}$is continuously differentiable.

In the proof below, it will be useful to adopt the viewpoint expressed in Sect. 1.3, i.e. to track the movements of the injected energies through time. Notice that for a sample path with no multiple collisions, there is no ambiguity whatsoever about the trajectory of an injected energy.

Proof: We verify that $\frac{\partial \eta_{i}}{\delta \varepsilon_{j}}, \frac{\partial \xi_{i}}{\delta \varepsilon_{j}}$ and $\frac{\partial x_{i}}{\delta \varepsilon_{j}}$ exist and are continuous on $\mathcal{E}$. For $\eta_{i}$ and $\xi_{i}$, it is easy: either $\varepsilon_{j}$ is carried by the particle in question, or it is not. If it is, then the partial derivative is $=1$; if not, then it is $=0$.

For $x_{i}$, consider first the case where $\eta_{i}$ carries the injection $\varepsilon_{k}, k \neq j$. We let $p$ be the total number of times $\varepsilon_{j}$ crosses the interval $[i, i+1]$ (in either direction) before 
$X_{n}$. If $p=0$, then $\frac{\partial x_{i}}{\delta \varepsilon_{j}}=0$. Suppose not. Then perturbing (only) $\varepsilon_{j}$ to $\varepsilon_{j}+\delta \varepsilon$, the time gained per crossing (with a sign) will be $\frac{1}{\sqrt{\varepsilon_{j}}}-\frac{1}{\sqrt{\varepsilon_{j}+\delta \varepsilon}}$. Consequently

$$
\frac{\partial x_{i}}{\delta \varepsilon_{j}}=\lim _{\delta \varepsilon \rightarrow 0} p \sqrt{\varepsilon_{k}} \cdot \frac{\frac{1}{\sqrt{\varepsilon_{j}}}-\frac{1}{\sqrt{\varepsilon_{j}+\delta \varepsilon}}}{\delta \varepsilon}=\frac{p}{2} \varepsilon_{k}^{\frac{1}{2}} \varepsilon_{j}^{-\frac{3}{2}} .
$$

The case where $\varepsilon_{j}$ is carried by $\eta_{i}$ is left to the reader.

In the setting above, if $D \Phi(\underline{\varepsilon})$ is onto as a linear map, then by the implicit function theorem, there is an open set $\mathcal{E}^{\prime} \subset \mathcal{E}$ with $\underline{\varepsilon} \in \mathcal{E}^{\prime}$ such that $\Phi$ carries Lebesgue measure on $\mathcal{E}^{\prime}$ to a measure in $\mathcal{M}^{+}$with a strictly positive density on a neighborhood of $\Phi(\underline{\varepsilon})$. This implies in particular $\left(p_{X_{0}}^{n}\right)_{a c}>0$. We summarize as follows:

Definition 3.1 We say $X \in \mathcal{M}^{+}$has full rank ${ }^{5}$ if there is a sequence of injections $\underline{\varepsilon}=\left(\varepsilon_{1}, \cdots, \varepsilon_{m}\right)$ leading to a sample path $\underline{X}=\left(X_{0}, X_{1}, \ldots, X_{n}\right)$ with $X_{0}=X$ such that (i) $\underline{X}$ has no multiple collisions, and (ii) $D \Phi_{X_{0}}(\underline{\varepsilon})$ is onto.

Corollary 3.1 If $X \in \mathcal{M}^{+}$has full rank and $\underline{X}$ is as above, then $p_{X}^{n}$ has strictly positive densities on an open set containing $X_{n}$.

Having full rank is obviously an open condition, meaning if $X$ has full rank, then so does $Y$ for all $Y$ sufficiently close to $X$.

\section{(B) Ergodic components}

One way to force two points to be in the same ergodic component (in the sense to be made precise) is to show that they have "overlapping futures". This motivates the following relation:

Definition 3.2 For $X, Y \in \mathcal{M}^{+}$, we write $X \sim Y$ if there exist a positive Lebesgue measure set $\mathcal{A}=\mathcal{A}(X, Y) \subset \mathcal{M}^{+}$and $m, n \in \mathbb{Z}^{+}$such that $\left.\left(p_{X}^{m}\right)_{\text {ac }}\right|_{\mathcal{A}}$ and $\left.\left(p_{Y}^{n}\right)_{\text {ac }}\right|_{\mathcal{A}}$ have strictly positive densities.

Here is how this condition will be used: Suppose $\mu$ and $\nu$ are ergodic measures, and there is a positive $\mu$-measure set $\mathcal{A}_{\mu}$ and a positive $\nu$-measure set $\mathcal{A}_{\nu}$ such that $X \sim Y$ for all $X \in \mathcal{A}_{\mu}$ and $Y \in \mathcal{A}_{\nu}$. Then the ergodic theorem tells us that $\mu=\nu$ since they have the same ergodic averages along positive measure sets of sample paths.

As an immediate corollary of the ideas in Part (A), we obtain

Corollary 3.2 If $X \in \mathcal{M}^{+}$has full rank, then there is a neighborhood $\mathcal{N}$ of $X$ such that $Y \sim Z$ for all $Y, Z \in \mathcal{N}$.

\footnotetext{
${ }^{5}$ This property has the flavor of Hörmander's condition for hypoellipticity for SDEs - in a setting that is largely deterministic and has discontinuities.
} 


\section{(C) Constant energy configurations}

For fixed $e \in(0, \infty)$, let

$$
\mathcal{Q}_{e}=\left\{X \in \mathcal{M}^{+}: \eta_{i}=\xi_{i}=e \text { for all } i\right\} .
$$

If we start from $X \in \mathcal{Q}_{e}$ and inject only energies having value $e$, then the resulting sample path(s) will remain in the set $\mathcal{Q}_{e}$. (Obviously, this is feasible only if $e \in$ $I_{L} \cap I_{R}$.) We call these constant-energy sample paths, and say $X \in \mathcal{Q}_{e}$ has no multiple collisions if that is true of its constant-energy sample path.

Needless to say, constant-energy sample paths occur with probability zero. However, they have very simple dynamics, and perturbations are relatively easy to control. Our plan is to exploit these facts by driving all sample paths to some $\mathcal{Q}_{e}$ and to work from there.

\subsection{Intermediate propositions}

We claim that Theorem 1 follows readily from the following two propositions:

Proposition 3.1 Every $X \in \mathcal{M}^{+}$eventually acquires a density.

Proposition $3.2 X \sim Y$ for a.e. $X, Y \in \mathcal{M}^{+}$with respect to Lebesgue measure.

We remark that to rule out the presence of singular invariant measures, we need Proposition 3.1 to hold for every $X$, not just almost every $X$.

Proof of Theorem 1 assuming Propositions 3.1 and 3.2: Proposition 3.1 and Lemma 3.1 together imply that every invariant probability measure has a density, proving part (b). To prove part (a), let $\mu$ and $\nu$ be ergodic measures. Since they have densities, Proposition 3.2 implies, as noted in Paragraph (B), that $\mu=\nu$.

Next we identify three (more concrete) conditions that will imply Propositions 3.1 and 3.2. The following shorthand is convenient: For $X, Y \in \mathcal{M}^{+}$, we write

$X \Rightarrow Y$ if given any neighborhood $\mathcal{N}$ of $Y$, there exists $n$ such that $p_{X}^{n}(\mathcal{N})>0$,

$X \Rightarrow Y$ if given any neighborhood $\mathcal{N}$ of $Y$, there is a neighborhood $\mathcal{N}^{\prime}$ of $X$ such that $X^{\prime} \Rightarrow Y$ for all $X^{\prime} \in \mathcal{N}^{\prime}$.

Proposition 3.3 Given $X \in \mathcal{M}^{+}$and $e \in I_{L} \cap I_{R}$, there exists $Z=Z(X) \in \mathcal{Q}_{e}$ such that $X \Rightarrow Z$.

Proposition $3.4 Z \Rightarrow Z^{\prime}$ for all $Z, Z^{\prime} \in \mathcal{Q}_{e}$, any $e \in I_{L} \cap I_{R}$.

Proposition 3.5 Every $Z \in \mathcal{Q}_{e}$ with no multiple collisions has full rank. 
Proofs of Propositions 3.1-3.2 assuming Propositions 3.3-3.5:

To prove Proposition 3.1, we concatenate Propositions 3.3 and 3.4 to show that for every $X \in \mathcal{M}^{+}, X \Rightarrow Z$ for some $Z=Z(X) \in \mathcal{Q}_{e}$ with no multiple collisions. The assertion is proved if there is a neighborhood $\mathcal{N}_{Z}$ of $Z$ such that every $Z^{\prime} \in \mathcal{N}_{Z}$ eventually acquires a density. This follows from Proposition 3.5, Corollary 3.1 and the fact that full rank is an open condition.

To prove Proposition 3.2, it is necessary to produce a common $Z=Z(X, Y) \in$ $\mathcal{Q}_{e}$ with no multiple collisions such that $X, Y \Rightarrow Z$. To this end, we first apply Proposition 3.3 to produce $Z(X) \in \mathcal{Q}_{e}$ with $X \Rightarrow Z(X)$ and $Z(Y) \in \mathcal{Q}_{e}$ with $Y \Rightarrow Z(Y)$. We then fix an (arbitrary) $Z \in \mathcal{Q}_{e}$ with no multiple collisions. Since $Z(X), Z(Y) \Rightarrow Z$ (Proposition 3.4), we have $X, Y \Rightarrow Z$ as before. Proposition 3.5 and Corollary 3.2 then give the desired result.

Proving Theorem 1 has thus been reduced to proving Propositions 3.3-3.5.

\section{Proofs of Propositions 3.3-3.5}

In Sects. 4.1-4.2, we give an algorithm for driving sample paths from given initial conditions to constant-energy surfaces. This can be viewed as changing the energies in a configuration. In Sects. 4.3-4.4, we focus on changing the relative positions of the moving particles.

\subsection{Sample paths from $X$ to $\mathcal{Q}_{e}$ ("typical" initial conditions)}

The hypothesis of Theorem 1 guarantees the existence of some $\xi \in I_{L} \cap I_{R}$. We may assume, without loss of generality, that $\xi=1$. Given $X \in \mathcal{M}^{+}$, we seek sample paths that lead to the constant energy surface $\mathcal{Q}_{1}$. Consider first the following specific question: By injecting only particles with energy 1, will we eventually "flush out" all the energies in $X$, replacing them with new particles having energy 1 ?

To study this question, we propose to suppress some information, to focus on the following evolution of arrays of energies: To each $X \in \mathcal{M}^{+}$, we associate the array $E(X)=\left(\eta_{0}, \xi_{1}, \eta_{1}, \xi_{2}, \cdots, \xi_{N}, \eta_{N}\right)$ where $\eta_{i}$ and $\xi_{i}$ are the energies of $X$ and they are arranged in the order shown. Then corresponding to each sample path $\underline{X}=\left(X_{0}, X_{1}, \cdots\right)$ is the sequence of moves $E\left(X_{0}\right) \rightarrow E\left(X_{1}\right) \rightarrow E\left(X_{2}\right) \rightarrow \cdots$. For example, collision between site $i$ and the particle on its right corresponds to swapping the $(2 i+1)$ st entry in the array with the $(2 i)$ th; the rightmost particle exiting the system and an energy of value $\xi_{R}$ entering corresponds to replacing the $(2 N+1)$ st entry by $\xi_{R}$, and so on. For as long as there are no same-site double collisions, we can trace the movements of energies in $\left(E\left(X_{0}\right), E\left(X_{1}\right), \cdots\right)$ as discussed in Sect. 1.3. We define the exit time of the $j$ th entry in $E\left(X_{0}\right)$ to be the number of moves before this energy exits the system, and define $T(\underline{X})$ to be the last exit time of all the elements in $E\left(X_{0}\right)$. A priori, $T(\underline{X}) \leq \infty$. 
Lemma 4.1 Given $X_{0} \in \mathcal{M}^{+}$, suppose that injecting all 1 s gives rise to a sample path $\underline{X}$ with no same-site double collisions. Then $T(\underline{X})<\infty$.

At each step, we will refer to the original energies of $X_{0}$ that remain as the "old energies", and the ones that are injected as "new energies". In particular, all new energies have value 1 (some old energies may also have value 1 ). The lemma asserts that in finite time, the number of old energies remaining will decrease to 0.

Proof: For each $n$, we let $E\left(X_{n}\right)=\left(\eta_{0}, \xi_{1}, \eta_{1}, \xi_{2}, \cdots, \xi_{N}, \eta_{N}\right)$, and say $X_{n}$ is in state

$$
\begin{aligned}
& (\cdot, i) \text { if the leftmost old energy is } \xi_{i}, \\
& (+, i) \text { if the leftmost old energy is } \eta_{i} \text { and } \sigma_{i}=+ \\
& (-, i) \text { if the leftmost old energy is } \eta_{i} \text { and } \sigma_{i}=-.
\end{aligned}
$$

The terminology above is not intended to suggest that we are working with a reduced system. No such system is defined; nevertheless it makes sense to discuss which transitions among these states are permissible in the underlying Markov dynamics. Notice first that independent of the state of a system, it will change eventually. This is because all energies are nonzero, so a collision involving the leftmost old energy is guaranteed to occur at some point. We list below all the transitions between states that are feasible, skipping over (many) steps in the Markov chain that do not involve the leftmost old energy:

(1) Suppose the system is in state $(-, i)$. If $i \neq 0$, then the only possible transition is $(-, i) \rightarrow(\cdot, i)$. If $i=0$, then the leftmost old energy exits the system, and the new state is determined by the next leftmost old energy (if one remains).

(2) Suppose the system is in state $(+, i)$. If $i=N$, then $T(\underline{X})$ is reached as this last remaining old energy exits the system. If not, we claim the only two possibilities are $(+, i) \rightarrow(\cdot, i+1)$ and $(+, i) \rightarrow(-, i)$. The first case corresponds to the energy originally at site $i+1$ being new, the second case old.

(3) Finally, consider the case where the system is in state $(\cdot, i)$. If the next collision is with the particle from the left, then $(\cdot, i) \rightarrow(-, i-1)$. If it is with the particle from the right, then there are two possibilities corresponding to the approaching energy being new or old, namely $(\cdot, i) \rightarrow(+, i)$ or $(\cdot, i) \rightarrow(\cdot, i)$. Notice that the system cannot remain in state $(\cdot, i)$ forever, since the particle from the left will arrive sooner or later, causing the state to change. For this reason, let us agree not to count $(\cdot, i) \rightarrow(\cdot, i)$ as a transition.

To summarize, the only possible transitions are

$$
(-, i) \rightarrow(\cdot, i), \quad(\cdot, i) \rightarrow(-, i-1),(+, i), \quad(+, i) \rightarrow(\cdot, i+1),(-, i)
$$

except where the transition leads to an old energy exiting the system. When that happens, either there is no old energy left, or the system can start again in any state. The following observation is crucial: 
Sublemma 4.1 The transition sequence $(-, i) \rightarrow(\cdot, i) \rightarrow(+, i)$ is forbidden.

We first complete the proof of Lemma 4.1 assuming the result in this sublemma: Observe first that if one starts from $(+, i)$, either the evolution is $(+, i) \rightarrow(\cdot, i+1) \rightarrow$ $(+, i+1) \rightarrow(\cdot, i+2) \rightarrow \cdots$ leading to an exit at the right, or a state of the form $(-, j)$ is reached; a similar assertion holds if one starts from $(\cdot, i)$. On the other hand, starting from $(-, i)$, the only possible sequence permitted by the sublemma is $(-, i) \rightarrow(\cdot, i) \rightarrow(-, i-1) \rightarrow(\cdot, i-1) \rightarrow \cdots$ leading to an exit at the left. This proves that in finite time (meaning a finite number of steps with respect to the Markov chain $p^{n}$ ), the number of old energies remaining will decrease by one.

Proof of Sublemma: Suppose the transition $(-, i) \rightarrow(\cdot, i)$ takes place at $t=0$. Since all the energies to the left of site $i$ are new and therefore have speed one, a particle from the left is guaranteed to reach site $i$ at time $t=t_{0}<2$, since the previous collision with the site from the left took place strictly before $t=0$ (no same-site double collisions). We will argue that when this particle arrives, it will find an old energy at site $i$, that in fact the state of the system has not changed between $t=0$ and $t_{0}$. Thus the next transition has to be $(\cdot, i) \rightarrow(-, i-1)$.

Here are the events that may transpire between $t=0$ and $t_{0}$ on the segment $[i, i+1]$ : Observe that the transition at $t=0$ is necessarily between an old and a new energy (otherwise the state at $t=0^{-}$could not be $(-, i)$ ). This will result in a new energy leaving site $i$ for site $i+1$ at $t=0^{+}$, and arriving at $t=1$. The only way $(\cdot, i) \rightarrow(+, i)$ can happen is for a new energy to move leftward on $[i, i+1]$, and to arrive at site $i$ before time $t_{0}$. This cannot happen, since new energies travel at unit speed. (Notice that the old energy at site $i$ may change between $t=1$ and $t_{0}$, but the state of the system does not.)

Partial proof of Proposition 3.3: We prove the result for $X=X_{0}$ under the additional assumption that injecting 1 s gives rise to a sample path with no same-site double collisions. By Lemma 4.1 , there is a sample path $\underline{X}=\left(X_{0}, \cdots, X_{n}\right)$ with $X_{n} \in \mathcal{Q}_{1}$. Let $\mathcal{N}$ be a neighborhood of $X_{n}$. If $\underline{X}$ has no multiple collisions, then all nearby sample paths will end in $\mathcal{N}$ as discussed in Sect. 3.1. If $\underline{X}$ has multiple (but not same-site) collisions, ${ }^{6}$ then injecting a slightly perturbed energy sequence may - is likely to, in fact - desynchronize the simultaneous jumps. If, for example, two collisions at sites $i$ and $i^{\prime}$ occur simultaneously at step $j$, then for perturbed injected energies, $X_{j}$ may be replaced by $X_{j}^{\prime}$ and $X_{j}^{\prime \prime}$ corresponding to two collisions that happen in quick succession. This aside, the situation is similar to that with no multiple collisions, and we still have $p_{X}^{k}(\mathcal{N})>0$ but possibly for some $k>n$.

\footnotetext{
${ }^{6}$ These points are discontinuities only for the (discrete-time) Markov chain, affecting the number of steps. They are not discontinuities at all for the continuous-time jump process.
} 


\subsection{Sample paths from $X$ to $\mathcal{Q}_{e}$ ("exceptional" cases)}

As noted in Sect. 3.2, to rule out the existence of singular invariant measures, it is necessary to show that every $X \in \mathcal{M}^{+}$eventually acquires a density. Our strategy is to inject energy 1s into the system and argue that this produces a sample path $\underline{X}$ that leads to a point $Z \in \mathcal{Q}_{1}$. To prove $X \Rightarrow Z$, however, requires more than that: it requires that a positive measure set of sample paths starting from $X$ follow $\underline{X}$. This is not a problem if $\underline{X}$ has no same-site double collisions, for in the absence of such collisions, the dynamics are essentially continuous (as explained above). Yet it is unavoidable that for some $X \in \mathcal{M}^{+}$, injecting 1s will lead to same-site double collisions. It is an exceptional situation, but one that we must deal with if we are to follow the same route of proof.

We now address the potential problems. Recall that at each same-site double collision, there are two ways to continue the sample path.

\section{Partitioning nearby sample paths according to itineraries}

Let $\underline{X}=\left(X_{0}, X_{1}, \cdots, X_{n}\right)$ be a sample path obtained by injecting a sequence of $m$ energies all of which are 1 s and making a specific choice at each same-site double collision. As in Sect. 3.1(A), we consider energies $\underline{\varepsilon}=\left(\varepsilon_{1}, \ldots, \varepsilon_{m}\right)$ near $\underline{1}=(1, \cdots, 1)$, and ask the following question: Which injection sequence $\underline{\varepsilon}$ will give rise to a sample path $\underline{X}^{(\varepsilon)}$ that has the same collision sequence as $\underline{X}$ ? To focus on the issues at hand, we will ignore multiple collisions that do not involve same-site double collisions, for they are harmless as explained earlier.

The computation in Lemma 3.2 motivates the following coordinate change, which is not essential but simplifies the notation: Let $\psi(\varepsilon)=\frac{1}{\sqrt{\varepsilon}}-1$, and let $\Psi\left(\varepsilon_{1}, \cdots, \varepsilon_{m}\right)=$ $\left(\psi\left(\varepsilon_{1}\right), \cdots, \psi\left(\varepsilon_{m}\right)\right)$. Then $\Psi$ maps a neighborhood of $\underline{1}$ diffeomorphically onto a neighborhood of $\underline{0}=(0, \cdots, 0)$. We assume that $\underline{X}^{(\underline{\varepsilon})}$ has the same itinerary as $\underline{X}$ through step $i-1$, and that at step $i, \underline{X}$ has a double collision at site $j$. To determine whether the left or right particle will arrive first at site $j$ for $\underline{X}^{(\varepsilon)}$, we use $\underline{X}$ as the point of reference, and let $t_{j}(\underline{\varepsilon})$ and $t_{j-1}(\underline{\varepsilon})$ be the times gained (with a sign) by the particles approaching site $j$ from the right and left respectively. Then

$$
t_{j}(\underline{\varepsilon})=p_{1} \psi\left(\varepsilon_{1}\right)+\cdots+p_{m} \psi\left(\varepsilon_{m}\right) \quad \text { and } \quad t_{j-1}(\underline{\varepsilon})=q_{1} \psi\left(\varepsilon_{1}\right)+\cdots+q_{m} \psi\left(\varepsilon_{m}\right)
$$

where $p_{k}$ and $q_{k}$ are the numbers of times - up to and including the approach to site $j$ - the injected energy $\varepsilon_{k}$ has passed through the intervals $[j, j+1]$ and $[j-1, j]$ respectively. Thus for $\underline{X}^{(\varepsilon)}$, the right particle arrives first if and only if $t_{j}(\underline{\varepsilon})>t_{j-1}(\underline{\varepsilon})$.

Thus given $\underline{X}=\left(X_{0}, X_{1}, \cdots, X_{n}\right)$ as above, there is a decreasing sequence of subsets $\mathbb{R}^{m}=V_{0} \supset V_{1} \supset V_{2} \supset \cdots \supset V_{n}$ defined as follows: $V_{i}=V_{i-1}$ except where a same-site double collision occurs; when a same-site double collision occurs, say at site $j$, we let $V_{i}=V_{i-1} \cap H$ where $H=\left\{\underline{\varepsilon} \in \mathbb{R}^{m}: t_{j}(\underline{\varepsilon}) \geq t_{j-1}(\underline{\varepsilon})\right\}$ or $\left\{t_{j}(\underline{\varepsilon}) \leq t_{j-1}(\underline{\varepsilon})\right\}$ depending on whether we have chosen to let the right or left particle arrive first in $\underline{X}$. If there are multiple same-site double collisions, then we intersect with the half-spaces 
corresponding to all of them. The sequence of $V_{i}$ so obtained will have the property that all $\underline{\varepsilon}$ close enough to $\underline{1}$ with $\Psi(\underline{\varepsilon}) \in V_{n}$ give sample paths that have the same collision sequence as $\underline{X}$. In particular, if $V_{n}$ is nontrivial, meaning it has interior, then the set of $\underline{\varepsilon}$ for which $\underline{X}^{(\underline{\varepsilon})}$ shadows $\underline{X}$ will have positive measure. That is to say, $X_{0} \Rightarrow X_{n}$. In general, there is no guarantee that $V_{n}$ is nontrivial.

Supposing $V_{n}$ for a sample path is nontrivial, we say the choices made at step $n+1$ are viable if they lead to a nontrivial $V_{n+1}$. Observe that inductively, a viable choice can be made at each step, since one of the two half-spaces must intersect nontrivially $V_{n}$ from the previous step.

We first treat a special situation involving the same-site collision of three energies all of which are $1 \mathrm{~s}$. The setting is as above.

Sublemma 4.2 Suppose $V_{n}$ is nontrivial for a sample path $\underline{X}$, and at step $n+1$, the energy $\varepsilon_{k}$, which is $=1$, is involved in a same-site double collision with two other energies both of which are 1 s. Then the choice to have $\varepsilon_{k}$ arrive first is viable if $\varepsilon_{k}$ has the following history:

(i) its movement up to the collision above is monotonically from left to right;

(ii) all of its previous same-site double collisions are with two other energy 1 .

Proof: By (i), we have that in the collision at step $n+1, p_{k}=0$ and $q_{k}=1$. Choosing to have $\varepsilon_{k}$ arrive first corresponds to choosing the half-space $\left\{\Psi\left(\varepsilon_{k}\right) \geq G(\underline{\varepsilon})\right\}$ where $G(\underline{\varepsilon})$ is a linear combination of $\Psi\left(\varepsilon_{i}\right), i \neq k$. By (ii), all the other half-spaces in the definition of $V_{n}$ either does not involve $\varepsilon_{k}$ or is of the same type as the one above.

Completing the proof of Proposition 3.3: Given $X_{0} \in \mathcal{M}^{+}$, we now inject $1 \mathrm{~s}$ and make a choice at every same-site double collision with the aim of obtaining a sample path $X_{0}, X_{1}, \cdots, X_{n}$ with $X_{n} \in \mathcal{Q}_{1}$ and $X_{0} \Rightarrow X_{n}$. Our plan is to follow the scheme in Sect. 4.1 to reach $\mathcal{Q}_{1}$, and to make viable choices along the way.

The proof of Lemma 4.1 is based on the following observation: the leftmost old energy either moves right monotonically until it exits, or it turns around and starts to go left, and by Sublemma 4.1, once that happens it must move left monotonically until it exits. Revisiting the arguments, we see that with the exception of Sublemma 4.1, all statements in the proof of Lemma 4.1 hold for any sample path, with whatever choices made at double collisions. Moreover, the only way Sublemma 4.1 can fail is that at $t=0$, a double collision occurs at site $i$, we choose to have the left particle arrive first, and this is followed by another double collision at $t=2$ at which time we choose to have the right particle arrive first. It is only through these "two bad decisions" that the leftmost old energy can turn around and head right again.

The choices at same-site double collisions are arbitrary provided the following two conditions are met:

(i) The choice must be viable.

(ii) In the setting of Sublemma 4.2, we choose to have $\varepsilon_{k}$ arrive first. 
Following these rules, it suffices to produce $X_{n} \in \mathcal{Q}_{1}$ for some $n$; the nontriviality of $V_{n}$ follows from (i). Suppose, to derive a contradiction, that no $X_{n}$ ever reaches $\mathcal{Q}_{1}$, i.e., a set of old energies is trapped in the chain forever. Let $i_{0}$ be the leftmost site that it visits infinitely often. That means from some step $n_{0}$ on, the leftmost energy never ventures to the left of site $i_{0}$, but it returns to site $i_{0}$ infinitely many times, each time repeating the "two bad decisions" scenario above.

The situation to the left of site $i_{0}$ is as follows: ¿From time $n_{0}$ on, all the energies strictly to the left of this site have speed one. Thus by rule (ii), after a new energy enters from the left bath, it will march monotonically to the right until at least site $i_{0}$, whether or not it is involved in any same-site double collisions.

To complete the proof, consider a moment after time $n_{0}$ when the leftmost old energy arrives at site $i_{0}$. From the discussion above we know it has to be involved in a same-site double collision, and that the energy approaching from the left is new. By (ii), we choose to have the new energy arrive first, making the first "bad decision". But then in the collision at the same site 2 units of time later, again we choose to have the energy from the left arrive first, causing the old energy to move to site $i_{0}-1$ and contradicting the definition of site $i_{0}$.

\subsection{Moving about on constant energy surfaces}

This subsection focuses on getting from $Z \in \mathcal{Q}_{e}$ to $Z^{\prime} \in \mathcal{Q}_{e}$ for $e \in I_{L} \cap I_{R}$. In general, this cannot be accomplished by considering sample paths that lie on $\mathcal{Q}_{e}$ alone, since such sample paths are periodic as we will see momentarily. Instead we will make controlled excursions from $\mathcal{Q}_{e}$ aimed at returning to specific target points. As before, we assume $1 \in I_{L} \cap I_{R}$ and work with $\mathcal{Q}_{1}$.

\section{Circular tracks between sites}

It is often useful to represent the pair $\left(x_{i}, \sigma_{i}\right)$ by a single coordinate $z_{i}$ and to view the particle as making laps in a circle of length 2 . More precisely, if we represent this circle as $[0,2] / \sim$ with end points identified, then $z_{i}=x_{i}$ for $\sigma_{i}=+$ and $z_{i}=2-x_{i}$ when $\sigma_{i}=-$. A continuous-time sample path $Z(t), t \geq 0$, for the process described in Section 1 then gives rise to a curve

$$
Z^{*}(t)=\left(z_{0}(t), z_{1}(t), \cdots, z_{N}(t)\right) \in \mathbb{T}^{N+1}
$$

(We write $\mathbb{T}^{N+1}$ even though each factor has length 2.) For each $i, z_{i}(t)$ goes around the circle in uniform motion, changing speed only at $z_{i}=0$ and 1 . Accordingly, we denote by $\partial\left(\mathbb{T}^{N+1}\right)$ the set of points such that $z_{i}=0$ or $z_{i}=1$ for (at least) one $i$.

Asterisks will be used to signify the use of circular-track notation: A discretetime sample path $\underline{Z}=\left(Z_{0}, Z_{1}, \cdots\right)$ in the notation of previous sections corresponds to $\underline{Z}^{*}=\left(Z_{0}^{*}, Z_{1}^{*}, \cdots\right)$ with $Z_{i}^{*} \in \partial\left(\mathbb{T}^{N+1}\right)$. Returns of $\underline{Z}$ to $\mathcal{M}_{0}^{+}=\mathcal{M}^{+} \cap\left\{x_{0}=0\right\}$ translates into returns of $\underline{Z}^{*}$ to $\partial\left(\mathbb{T}^{N+1}\right) \cap\left\{z_{0}=0\right\}$. 
We now identify two features of constant energy configurations that make them easy to work with, beginning with the simplest case:

Lemma 4.2 Suppose we start from $Z \in \mathcal{Q}_{1}$ with no multiple collisions.

(a) By injecting all $1 \mathrm{~s}$, the sample path returns to $Z$ in $2 N+2$ steps.

(b) If all the injected energies are $1 s$ except possibly for one, which we call e, and if we assume the resulting sample path has no same-site double collisions, then the energy e moves monotonically through the system until it exits, after which the system rejoins the periodic sample path in (a).

Proof: (a) This is especially easy to see in continuous time and in circular-track notation: each $z_{i}(t)$ is periodic with period 2 . Thus the continuous-time sample path $Z(t)$ is periodic with period 2 , and in every two units of time, there are exactly $2 N+2$ collisions.

(b) Suppose the energy $e$ has just arrived at site $j$ from the left. Then the next collision at site $j$ is with the particle from the right, because all cycles take 2 units of time to complete and the right one has a (strict) headstart. This proves the monotonic movement of the energy $e$. After it leaves the system, the relative positions between $z_{i}$ and $z_{j}$ remain unchanged for all $i, j$, since the passage of the energy $e$ through the chain leads to identical time gains for $z_{i}$ and $z_{j}$.

Another nice property of constant energy sample paths is that the process is continuous along these paths. We make precise this idea:

Definition 4.1 Given a sample path $\underline{X}=\left(X_{0}, X_{1}, \ldots, X_{n}\right)$, we say the process is continuous at $\underline{X}$ if the following holds: Let $\underline{\varepsilon}=\left(\varepsilon_{1}, \ldots, \varepsilon_{m}\right)$ be the sequence in injections. Then given any neighborhood $\mathcal{N}$ of $X_{n}$, there are neighborhoods $\mathcal{N}_{0}$ of $X_{0}$ and $\mathcal{U}$ of $\underline{\varepsilon}$ such that for all $X_{0}^{\prime} \in \mathcal{N}_{0}$ and $\underline{\varepsilon}^{\prime} \in \mathcal{U}$, all the sample paths generated follows $\underline{X}$ into $\mathcal{N}$. (We do not require that it reaches $\mathcal{N}$ in exactly $n$ steps; it is likely to take more than $n$ steps if $\underline{X}$ has multiple collisions.)

Lemma 4.3 Let $\underline{X}=\left(X_{0}, \ldots, X_{n}\right)$ be a sample path.

(a) If $\underline{X}$ either has no same-site double collisions or every such collision involves three identical energies, then the process is continuous at $\underline{X}$.

(b) If the process is continuous at $\underline{X}$, then $X_{0} \Rightarrow X_{n}$.

The ideas behind part (a) are the same as those discussed in Sects. 4.1 and 4.2 and will not be repeated here. Part (b) is immediate, for " $\Rightarrow$ " requires less than continuity.

The following lemma is the analog of Lemma 4.2(b) without the assumption of "no multiple collisions": 
Lemma 4.4 Given any $Z \in \mathcal{Q}_{1}$, let all the energies injected be 1 s except possibly for one which has value e. Then (i) there is a sample path in which the energy e moves through the system monotonically; (ii) except for a discrete set of values e, it will do so without being involved in any same-site double collisions.

Proof: The monotonic motion of $e$ is clear if it is not involved in any same-site double collisions. If it is, choose to have it arrive first every time. This proves (i). To prove (ii), let $J$ be a finite interval of possible energies to be injected at $t=0$, and suppose $e$ is on its way from site 0 to site 1 . In circular-tracks notation, $z_{1}$ is periodic with period 2, and except for a finite subset of $e \in J, z_{0} \neq 1$ at exactly the same time that $z_{1}=0$. We avoid this "bad set" of $e$, ensuring that the special energy will not be in a double collision at site 1 . Now this energy leaves site 1 exactly when $z_{1}=0$, and at such a moment, the configuration to the right of site 1 is identical (independently of the value of $e$ in use). The same argument as before says that to avoid a double collision at site 2 , another finite subset of $J$ may have to be removed.

Injecting a single energy different than 1 will not produce the desired positional variations. We now show that injecting a second energy - both appropriately chosen and injected at appropriate times - will do the trick. The hypothesis of Theorem 1 guarantees, after scaling to put $1 \in I_{L} \cap I_{R}$, that there exists $e^{\prime} \in I_{L} \cup I_{R}$ with $e^{\prime}=o\left(1 / N^{2}\right)$. (Lemma 4.5 below is the only part of the proof in which this hypothesis is used.) Let us assume for definiteness that $e^{\prime} \in I_{L}$, and consider initial conditions $Z \in \mathcal{M}_{0}^{+}=\mathcal{M}^{+} \cap\left\{x_{0}=0\right\}$.

Lemma 4.5 There exists $a_{0}>0$ for which the following hold: Given a with $|a|<a_{0}$, $Z_{0} \in \mathcal{Q}_{1} \cap \mathcal{M}_{0}^{+}$and $k \in\{1, \ldots, N\}$, there is a sample path $\underline{Z}=\left(Z_{0}, \cdots, Z_{n}\right)$ with $Z_{n} \in \mathcal{Q}_{1} \cap \mathcal{M}_{0}^{+}$such that if $Z_{0}^{*}=\left(0, z_{1}, \ldots, z_{N}\right)$, then

$$
Z_{n}^{*}=\left(0, z_{1}, \ldots, z_{k-1}, z_{k}-a, z_{k+1}-a, \ldots, z_{N}-a\right)
$$

Moreover, the sample path $\underline{Z}$ may be chosen so that it has no same-site double collisions except where all the energies involved are $1 \mathrm{~s}$.

Proof: First we focus on constructing a sample path that leads to the desired $Z_{n}$ without attempting to avoid double collisions.

In the construction to follow, all the injections except for two will be 1s. At $t=0$, we inject energy $e^{\prime}=o\left(1 / N^{2}\right)$ from the left bath, letting it move monotonically from left to right. When it is about halfway between sites $k$ and $k+1$, we inject from the left an energy $e=e(a) \approx 1$ (the relation between $e$ and $a$ will be clarified later). The energy $e$ also moves monotonically from left to right until it reaches the $k$ th site, which takes $O(N)$ units of time. Since the slow particle takes longer than $O(N)$ units of time to reach site $k+1$, the $e$-energy waiting at site $k$ is met by a particle from the left. So it turns around and moves monotonically left, eventually exiting at the left 
end. As for the slow energy, it eventually reaches site $k+1$, and continues its way monotonically to the right until it exits the chain.

Let $Z_{n}$ be the first return to $\mathcal{M}_{0}^{+}$after the exit of $e^{\prime}$ (the $e$-energy exits much earlier). By definition, $Z_{n} \in \mathcal{Q}_{1}$. It remains to investigate its $z_{i}$-coordinates. Reasoning as in the proof of Lemma 4.2, we see that the $e^{\prime}$-energy does not cause a shift in the relative positions of the $z_{i}$ because its effects on all of the $z_{i}$ are identical. The $e$-energy, on the other hand, has made one full lap (first half on its way to site $k$ and second half on its way back) on the segments $[j, j+1]$ for $j=0,1, \cdots, k-1$ but not for $j \geq k$. Let $a=2\left(1-e^{-\frac{1}{2}}\right)$ be the (signed) time gain per lap for a particle with energy $e$ over a particle with energy 1 . Then the position of $z_{0}$ relative to $z_{j}$ for $j<k$ is maintained while relative to $z_{j}$ for $j \geq k, z_{0}$ is ahead by distance $a$, leading to the coordinates of $Z_{n}$ as claimed.

We now see that the bound imposed on $a$ is that $e(a)$ must be in $I_{L} \cap I_{R}$. This completes the proof of the lemma except for the claim regarding double collisions.

From Lemma 4.4(b), we know that for $e^{\prime}$ outside of a discrete set of values, the particle carrying this special energy will not be involved in any same-site double collisions. It remains to arrange for the energy $e$ to avoid such collisions. Here the situation is different: since $a$ is given, $e$ is fixed, but notice that increasing $e^{\prime}$ is tantamount to delaying the injection of the energy $e$. Thus an argument similar to that in Lemma 4.4(b) can be used to show that by avoiding a further discrete set of values for $e^{\prime}$ (depending on $Z_{0}, e$ and $k$ ), the energy $e$ will not be involved in same-site double collisions. This completes the proof.

Remark. The following two facts make this result very useful: (a) The dynamical events described in Lemma 4.5 require no pre-conditions on $Z_{0}$ other than $Z_{0} \in$ $\mathcal{Q}_{1} \cap \mathcal{M}_{0}^{+}$. (b) The range of admissible $a$ is independent of $Z_{0}$.

Suppose $a>0$, and for some $j>k$, the $j$ th moving particle in $Z_{0}$ is such that $x_{j}<a$ and $\sigma_{j}=+$. Then it will follow that $\sigma_{j}=-$ in $Z_{n}$, since it is distance $a$ behind its original position. This is very natural when one thinks about it in terms of circular tracks, but it is a jump in the phase space topology in Sect. 1.1.

\subsection{Proofs of Propositions 3.4 and 3.5}

Proof of Proposition 3.4: Given $Z, Z^{\prime} \in \mathcal{Q}_{1}$, we will construct a sample path $\underline{Z}=\left(Z_{0}, Z_{1}, \cdots, Z_{n}\right)$ with $Z_{0}=Z, Z_{n}=Z^{\prime}$ and $Z_{0} \Rightarrow Z_{n}$. Since all constant energy sample paths pass through $\mathcal{M}_{0}^{+}$, we may assume $Z, Z^{\prime} \in \mathcal{M}_{0}^{+}$.

Let $Z^{*}=\left(0, z_{1}, \cdots, z_{N}\right)$ and $\left(Z^{\prime}\right)^{*}=\left(0, z_{1}^{\prime}, \cdots, z_{N}^{\prime}\right)$. Our plan is to apply Lemma 4.5 as many times as needed to nudge each $z_{i}$ toward $z_{i}^{\prime}$ one $i$ at a time beginning with $i=1$. Write $z_{1}-z_{1}^{\prime}=j a$ for some $j \in \mathbb{Z}^{+}$and $a$ small enough for Lemma 4.5. Applying Lemma $4.5 j$ times with $k=1$, we produce $Z_{n_{1}} \in \mathcal{Q}_{1} \cap \mathcal{M}_{0}^{+}$with

$$
Z_{n_{1}}^{*}=\left(0, z_{1}^{\prime}, z_{2}+\left(z_{1}^{\prime}-z_{1}\right), \cdots, z_{n}+\left(z_{1}^{\prime}-z_{1}\right)\right)
$$


We then apply Lemma 4.5 to $Z_{n_{1}}$ with $k=2$ repeatedly to change it to $Z_{n_{2}}$ with

$$
Z_{n_{2}}^{*}=\left(0, z_{1}^{\prime}, z_{2}^{\prime}, z_{3}+\left(z_{2}^{\prime}-z_{2}-\left(z_{1}^{\prime}-z_{1}\right)\right), \cdots, z_{n}+\left(z_{2}^{\prime}-z_{2}-\left(z_{1}^{\prime}-z_{1}\right)\right)\right),
$$

and so on until $Z_{n}=Z_{n_{N}}$ is reached.

Notice that the sample path $\underline{Z}$ so obtained has the property that it has no same-

site double collisions other than those that involve three identical energies (all of which $1 \mathrm{~s}$ ). Lemma 4.3 says that the dynamics are continuous at such sample paths. Hence $Z \Rightarrow Z^{\prime}$.

Proof of Proposition 3.5: Given $Z \in \mathcal{Q}_{1}$ with no multiple collisions, our aim here is to produce a sample path $Z_{0}=Z, Z_{1}, \cdots, Z_{n}$, also free of multiple collisions, by injecting a sequence of energies $\underline{\varepsilon}=\left(\varepsilon_{1}, \cdots, \varepsilon_{m}\right)$ so that in the notation of Sect. 3.1, the map $\Phi$ has the property that $D \Phi(\underline{\varepsilon})$ is onto. We first give the algorithm, with explanations to follow:

(1) We inject 1s until $n_{0}$, the first time $Z_{n_{0}} \in \mathcal{M}_{0}^{+}$.

(2) At step $n_{0}$, we inject into the system an energy $e^{\prime}=o\left(1 / N^{2}\right)$ chosen to avoid multiple collisions, followed by all 1s, until the first time the sample path returns to $\mathcal{Q}_{1} \cap \mathcal{M}_{0}^{+}$; call this step $n_{1}$.

(3) By Lemma 4.1, $T\left(Z_{n_{1}}\right)<\infty$. Let $n$ be the smallest integer $\geq n_{1}+T\left(Z_{n_{1}}\right)$ for which $Z_{n} \in \mathcal{M}_{0}^{+}$.

We now prove that $D \Phi(\underline{\varepsilon})$ has full rank:

Let $\delta \eta_{i}$ denote an infinitesimal displacement in the $\eta_{i}$ variable at $Z_{n}$. To see that $\delta \eta_{i}$ is in the range of $D \Phi(\underline{\varepsilon})$, we trace this energy backwards (in the sense of Sect. 4.1) to locate its point of origin. (3) above ensures that it was injected at step $j$ for some $n_{1}<j \leq n$. Varying $\varepsilon_{j}$, therefore, leads directly to variations in $\eta_{i}$. The same argument applies to $\delta \xi_{i}$.

Next we consider displacements in $x_{i}$. Assume for definiteness that $e^{\prime}$ enters the system from the left. We fix $k \in\{1, \cdots, N\}$, and let $\varepsilon_{j}, j=j(k)$, be one of the energies injected from the left when $e^{\prime}$ is about halfway between sites $k$ and $k+1$. By Lemma 4.5, varying $\varepsilon_{j}$ leads to a variation of the form $\delta_{k}=\delta x_{k}+\cdots+\delta x_{N}$ in $Z_{n_{1}}$. By Lemma 4.2, this displacement is retained between $Z_{n_{1}}$ and $Z_{n}$ since only 1 s are injected. The vectors $\left\{\delta_{k}, k=1,2, \cdots, N\right\}$ span the subspace corresponding to positional variations.

The proof of Proposition 3.5 is complete.

\section{$5 \quad$ Equilibrium Distributions}

\subsection{Systems with a single site}

This subsection treats exclusively the case $N=1$. As we will see, nearly all of the ideas in a complete proof of Theorem 2 (for general $N$ ) show up already in this very 
simple situation. Following the notation in Sect. 1.1, we consider phase variables

$$
X=\left(\eta_{0}, \xi, \eta_{1} ; x_{0}, x_{1} ; \sigma_{0}, \sigma_{1}\right) \in(0, \infty)^{3} \times[0,1]^{2} \times\{-,+\}^{2}
$$

the phase space has 4 components corresponding to the 4 elements of $\{-,+\}^{2}$. Define

$$
\tau(\eta)=\frac{1}{Z} \frac{\rho(\eta)}{\sqrt{\eta}} \quad \text { where } \quad Z=\int_{0}^{\infty} \frac{\rho(\eta)}{\sqrt{\eta}} d \eta
$$

Theorem 2 asserts the invariance of the probability measure

$$
\mu=\tau\left(\eta_{0}\right) \rho(\xi) \tau\left(\eta_{1}\right) I_{[0,1]^{2}}\left(x_{0}, x_{1}\right) d \eta_{0} d \xi d \eta_{1} d x_{0} d x_{1} \times w_{4}
$$

where $I_{(\cdot)}$ is the indicator function and $w_{4}$ gives equal weight to the 4 points. Let $\mu_{t}=\int P_{X}^{t} d \mu(X)$ be the distribution at time $t$ with initial distribution $\mu$. To prove Theorem 2 , we need to show $\mu_{t}=\mu$ for all $t>0$, equivalently for all small enough $t>0$.

We say a system undergoes a simple change in configuration between times 0 and $t$ if during this period at most one collision occurs and this collision involves only a single moving particle. We will show in a sense to be made precise that for any system (independent of size), in short enough time intervals it suffices to consider simple changes in configuration. This observation simplifies the proof for $N=1$. It is crucial in the analysis of $N$-chains; without it, the complexity of the situation gets out of hand quickly. In stochastic processes, analogous ideas are provided by independence and exponential clocks. The dynamics being largely deterministic here, we will have to argue it "by hand", deducing it from tail assumptions on $\rho$.

In what follows, $|\nu|$ denotes the total variation norm of a finite signed measure $\nu$, and $\mu=\nu+O(t)$ means $|\mu-\nu|=O(t)$. For notational simplicity, we assume $\rho(\eta)=O\left(\eta^{-2}\right)$ as $\eta \rightarrow \infty$.

\section{Proof of Theorem 2 for the case $N=1$.}

\section{Step 1. Reduction to simple changes in configuration}

We will prove $\left|\mu_{t}-\mu\right|=O\left(t^{1+\delta}\right)$ for some $\delta>0$ as $t \rightarrow 0$. To see that this implies the invariance of $\mu$, fix $s>0$. By a repeated application of the estimate above with step size $\frac{s}{n}$, we obtain $\mu_{s}=\mu+n \cdot O\left(\left(\frac{s}{n}\right)^{1+\delta}\right)$, which tends to $\mu$ as $n \rightarrow \infty$.

In what follows, we focus on the component $M^{(+,+)}=\left\{\left(\sigma_{0}, \sigma_{1}\right)=(+,+)\right\}$; other components are analyzed similarly. Instead of comparing $\mu$ and $\mu_{t}$ directly on all of $M^{(+,+)}$, we will compare $\mu$ with some $\hat{\mu}_{t} \approx \mu_{t}$ (to be defined) on a large subset $\Gamma_{t} \subset M^{(+,+)}$(to be defined).

(A) We consider in the place of $\mu_{t}$ the measure $\hat{\mu}_{t}=\int P_{X}^{t} d \hat{\mu}(X)$ where $\hat{\mu}$ is the restriction of $\mu$ to the set $\left\{\eta_{0}, \xi, \eta_{1}<t^{-2}\right\}$. Notice that if all particles in a system have energy $<t^{-2}$, then their speeds are $<t^{-1}$, so no moving particle colliding with 
a site or a bath can set off a second collision to take place within $t$ units of time. We claim that confusing $\mu_{t}$ with $\hat{\mu}_{t}$ leads to an error of a size we can tolerate, since

$$
\int_{t^{-2}}^{\infty} \rho(\xi) d \xi=O\left(t^{2}\right) \quad \text { and } \quad \int_{t^{-2}}^{\infty} \tau(\eta) d \eta=O\left(t^{3}\right)
$$

(B) Next, we permit at most one moving particle to have a collision. For a particle at $x \in(0,1)$ with $\sigma=+$ and speed $\sqrt{\eta}$, a collision occurred in the previous $t$ units of time if and only if $t \sqrt{\eta}>x$. Let

$$
\Gamma_{t}=\left\{X \in M^{(+,+)}: \text {(i) } \eta_{0}, \xi, \eta_{1}<t^{-2} \text { and (ii) } t \sqrt{\eta_{0}}<x_{0} \text { or } t \sqrt{\eta_{1}}<x_{1}\right\} \text {. }
$$

We claim that $\mu\left(\Gamma_{t}\right)=1-O\left(t^{1+\delta}\right)$. This is because if $t \sqrt{\eta}>x$, then either (a) $\eta>t^{-\frac{2}{3}}$, or (b) $x<t \cdot t^{-\frac{1}{3}}=t^{\frac{2}{3}}$. Now (a) occurs with probability $\int_{t^{-\frac{2}{3}}}^{\infty} \tau(\eta) d \eta=O(t)$, and (b) occurs with probability $t^{\frac{2}{3}}$. Thus $\mu\left\{t \sqrt{\eta_{0}}>x_{0}\right.$ and $\left.t \sqrt{\eta_{1}}>x_{1}\right\}=\left[O\left(t^{\frac{2}{3}}\right)\right]^{2}=O\left(t^{\frac{4}{3}}\right)$.

The implications of (A) and (B) above are as follows: Suppose we show

$$
\left.(*) \quad \hat{\mu}_{t}\right|_{\Gamma_{t}}=\left.\mu\right|_{\Gamma_{t}}+O\left(t^{1+\delta}\right)
$$

for some $\delta$ with $0<\delta \leq \frac{1}{3}$. Then by (B), we have

$$
\mu=\mu \cdot I_{\Gamma_{t}}+O\left(t^{1+\delta}\right)=\hat{\mu}_{t} \cdot I_{\Gamma_{t}}+O\left(t^{1+\delta}\right) .
$$

Now we know from $(\mathrm{A})$ that $\left|\hat{\mu}_{t}\right|=1-O\left(t^{1+\delta}\right)$. This together with the equality above implies $\hat{\mu}_{t}\left(\Gamma_{t}^{c}\right)=O\left(t^{1+\delta}\right)$, so that $\hat{\mu}_{t} \cdot I_{\Gamma_{t}}=\hat{\mu}_{t}+O\left(t^{1+\delta}\right)$. Thus

$$
\mu=\hat{\mu}_{t}+O\left(t^{1+\delta}\right)=\mu_{t}+O\left(t^{1+\delta}\right)
$$

the second equality following from (A). This is what we seek.

Step 2. Analysis of simple changes in configuration

Let $\varphi$ and $\hat{\varphi}_{t}$ be the densities of $\mu$ and $\hat{\mu}_{t}$ respectively, and fix $\bar{X}=\left(\bar{\eta}_{0}, \bar{\xi}, \bar{\eta}_{1} ; \bar{x}_{0}, \bar{x}_{1}\right.$; $+,+) \in \Gamma_{t}$. We seek to compare $\varphi(\bar{X})$ and $\hat{\varphi}_{t}(\bar{X})$, remembering that to obtain $\hat{\mu}_{t}$, one considers only initial conditions in which all energies are $<t^{-2}$.

Case 1: $t \sqrt{\bar{\eta}_{0}}<\bar{x}_{0}$ and $t \sqrt{\bar{\eta}_{1}}<\bar{x}_{1}$. The only way to reach $\bar{X}$ in time $t$ is to start from $\left(\bar{\eta}_{0}, \bar{\xi}, \bar{\eta}_{1} ; \bar{x}_{0}-t \sqrt{\bar{\eta}_{0}}, \bar{x}_{1}-t \sqrt{\bar{\eta}_{1}} ;+,+\right)$. No collision occurs, and $\varphi(\bar{X})=\hat{\varphi}_{t}(\bar{X})$.

Case 2: $t \sqrt{\bar{\eta}_{0}}<\bar{x}_{0}$ and $t \sqrt{\bar{\eta}_{1}}>\bar{x}_{1}$. Let $s \sqrt{\bar{\eta}_{1}}=\bar{x}_{1}$. We claim that the only way to reach $\bar{X}$ in time $t$ is to start from $\left(\bar{\eta}_{0}, \xi, \eta_{1} ; \bar{x}_{0}-t \sqrt{\bar{\eta}_{0}}, x_{1} ;+,-\right)$ where $\xi=\bar{\eta}_{1}, \eta_{1}=\bar{\xi}$ and $x_{1}=(t-s) \sqrt{\eta_{1}}$. Starting here, one reaches $\bar{X}$ after a single exchange of energy between the right particle and the site. To compute the densities at $\bar{X}$, we let $\varepsilon>0$ be a very small number. Initial conditions with $x_{1} \in((t-s) \sqrt{\bar{\xi}}-\Delta,(t-s) \sqrt{\bar{\xi}}+\Delta)$ and $\Delta / \sqrt{\bar{\xi}}=\varepsilon / \sqrt{\bar{\eta}_{1}}$ will reach the target interval $\left(\bar{x}_{1}-\varepsilon, \bar{x}_{1}+\varepsilon\right)$ at time $t$. Since

$$
\rho\left(\bar{\eta}_{1}\right) \tau(\bar{\xi}) \Delta=\rho\left(\bar{\eta}_{1}\right) \frac{1}{Z} \frac{\rho(\bar{\xi})}{\sqrt{\bar{\xi}}} \Delta=\rho\left(\bar{\eta}_{1}\right) \frac{1}{Z} \frac{\rho(\bar{\xi})}{\sqrt{\bar{\eta}_{1}}} \varepsilon=\rho(\bar{\xi}) \tau\left(\bar{\eta}_{1}\right) \varepsilon,
$$


we conclude that $\varphi(\bar{X})=\hat{\varphi}_{t}(\bar{X})$.

Case 3: $t \sqrt{\bar{\eta}_{0}}>\bar{x}_{0}$ and $t \sqrt{\bar{\eta}_{1}}<\bar{x}_{1}$. Here we let $s$ be such that $s \sqrt{\bar{\eta}_{0}}=\bar{x}_{0}$. The only way to reach $\bar{X}$ is for the left particle to start at $x_{0}=(t-s) \sqrt{\eta_{0}}<1$ if $\eta_{0}$ is its initial energy, go left, reach the left bath at time $t-s$, and have the newly emitted energy $\bar{\eta}_{0}$ reach $\bar{x}_{0}$ at time $t$. To compare densities, again fix a small target interval $\left(\bar{x}_{0}-\varepsilon, \bar{x}_{0}+\varepsilon\right)$. This forces $x_{0} \in\left((t-s) \sqrt{\eta_{0}}-\Delta\left(\eta_{0}\right),(t-s) \sqrt{\eta_{0}}+\Delta\left(\eta_{0}\right)\right)$ with $\Delta\left(\eta_{0}\right) / \sqrt{\eta_{0}}=\varepsilon / \sqrt{\bar{\eta}_{0}}$. Thus

$$
\begin{aligned}
\hat{\varphi}_{t}(\bar{X}) & =\frac{1}{4} \rho(\bar{\xi}) \tau\left(\bar{\eta}_{1}\right) \cdot\left(\int_{0}^{t^{-2}} \tau\left(\eta_{0}\right) \frac{\sqrt{\eta_{0}}}{\sqrt{\bar{\eta}_{0}}} d \eta_{0}\right) \cdot \rho\left(\bar{\eta}_{0}\right) \\
& =\frac{1}{4} \rho(\bar{\xi}) \tau\left(\bar{\eta}_{1}\right) \cdot \frac{1}{Z} \frac{\rho\left(\bar{\eta}_{0}\right)}{\sqrt{\bar{\eta}_{0}}} \int_{0}^{t^{-2}} \rho\left(\eta_{0}\right) d \eta_{0}=\varphi(\bar{X}) \cdot\left(1-O\left(t^{2}\right)\right) .
\end{aligned}
$$

Since $t \sqrt{\bar{\eta}_{0}}>\bar{x}_{0}$ and $t \sqrt{\bar{\eta}_{1}}>\bar{x}_{1}$ is not permitted for $\bar{X} \in \Gamma_{t}$, we have exhausted all viable cases, completing the proof of $(*)$.

\subsection{Proof of Theorem 2}

We follow closely Sect. 5.1, adapting the ideas there to chains with $N$ sites.

We begin with the analogous set of reductions. As in Sect. 5.1, we seek to show $\mu_{t}=\mu+O\left(t^{1+\delta}\right)$ for some $\delta>0$, focusing on a fixed component of the phase space $M^{(\bar{\sigma})}=\left\{\left(\sigma_{0}, \cdots, \sigma_{N}\right)=\bar{\sigma}\right\}$. Instead of $\mu_{t}$, we consider $\hat{\mu}_{t}=\int P_{X}^{t} d \hat{\mu}(X)$ where $\hat{\mu}$ is the restriction of $\mu$ to $\left\{\eta_{i}, \xi_{i}<t^{-2}\right.$, all $\left.i\right\}$. It follows from the estimates in Sect. 5.1 that (for fixed $N$ ) this introduces an error within the tolerable range:

$$
\left|\mu_{t}-\hat{\mu}_{t}\right|<N \int_{t^{-2}}^{\infty} \rho+(N+1) \int_{t^{-2}}^{\infty} \tau=O\left(t^{2}\right)
$$

We define $\Gamma_{t}=\Gamma_{t}^{(\bar{\sigma})}$ to be

$$
\begin{aligned}
\left\{X \in M^{(\bar{\sigma})}:\right. & (i) \xi_{i}, \eta_{i}<t^{-2} \text { for all } i \\
& (\text { ii }) t \sqrt{\eta_{i}}<x_{i} \text { if } \sigma_{i}=+, t \sqrt{\eta_{i}}<1-x_{i} \text { if } \sigma_{i}=- \\
& \text { for all except at most one } i\}
\end{aligned}
$$

the idea being that when the above relation between $t, \sqrt{\eta_{i}}$ and $x_{i}$ holds, no collision occurs in the $t$ units of time prior to arrival in that configuration. The same argument as before shows that $\mu\left(\Gamma_{t}\right)=1-O\left(t^{\frac{4}{3}}\right)$ : the set on which this relation is violated by two of the moving particles has measure $O\left(t^{\frac{4}{3}}\right)$; the set on which it is violated by more than two particles is smaller.

As in Sect. 5.1, we seek to compare $\hat{\varphi}_{t}$ and $\varphi$ on the set $\Gamma_{t}$. As before, all changes in configuration involved are simple: there are 3 cases corresponding to no collisions, 
a collision with a site, and a collision with a bath. The analysis is identical to that in Sect. 5.1.

\section{PART II. NUMERICAL RESULTS}

We report here some results on nonequilibrium steady states (NESS) obtained by studying numerically two families of models: the first has exponential bath injections and the second, which we call the "two-energy model", is chosen for the transparency of the role played by the integrable dynamics.

\section{Exponential Baths}

We consider in this section processes defined in Sect. 1.1 for which the bath injections have exponential distributions, i.e., $L(\xi)=\beta_{L} e^{-\beta_{L} \xi}$ and $R(\xi)=\beta_{R} e^{-\beta_{R} \xi}$ where, as usual, $\beta_{L}=T_{L}^{-1}$ and $\beta_{R}=T_{R}^{-1}$ are to be thought of as inverse temperatures. We are interested in steady states of systems that are in contact with two baths at unequal temperatures, i.e., where $T_{L} \neq T_{R}$. It follows from Theorem 1 that all invariant distributions that arise from these bath injections are ergodic.

In Sect. 6.1, we demonstrate that mean energy profiles are well defined, both for finite $N$ and as $N \rightarrow \infty$. In Sect. 6.2, we focus on specific points along the chain, and investigate marginals of the NESS on (very) short segments. In the simulations shown, mean bath temperatures are $T_{L}=1$ and $T_{R}=10$, and chains of various lengths up to $N=1600$ are used.

\subsection{Macroscopic energy profiles}

For a chain with $N$ sites, we let $\mathbb{E}\left[\xi_{i}\right]$ denote the mean energy at site $i$, "mean" being taken with respect to the unique steady state distribution. Let $f_{N}:[0,1] \rightarrow \mathbb{R}$ be the function which linearly interpolates $f_{N}\left(\frac{i}{N+1}\right)=\mathbb{E}\left[\xi_{i}\right], i=1, \cdots, N$. If as $N$ increases, $f_{N}$ converges (pointwise) to a function $f$ on $[0,1]$, we will call $f$ the siteenergy profile of this model. Similarly, we let $f_{N}^{\sharp}$ be the function that interpolates $f_{N}^{\sharp}\left(\frac{i+1}{N+2}\right)=\mathbb{E}\left[\eta_{i}\right], \quad i=0,1, \cdots, N$, and call $f^{\sharp}=\lim _{N} f_{N}^{\sharp}$ the gap-energy profile. Profiles of gap energies conditioned on $\sigma_{i}$ being + or - are denoted $f_{N}^{+}$and $f_{N}^{-}$.

Fig. 1 shows plots of $f_{N}, f_{N}^{ \pm}$, and $f_{N}^{\sharp}$. Finite-chain profiles are found to vary little as $N$ goes from 100 to 1600, so we assume the limit profile will not be too different. Convergence time to steady state is slow, and increases with $N$ as expected. (See numerical details in caption.) 


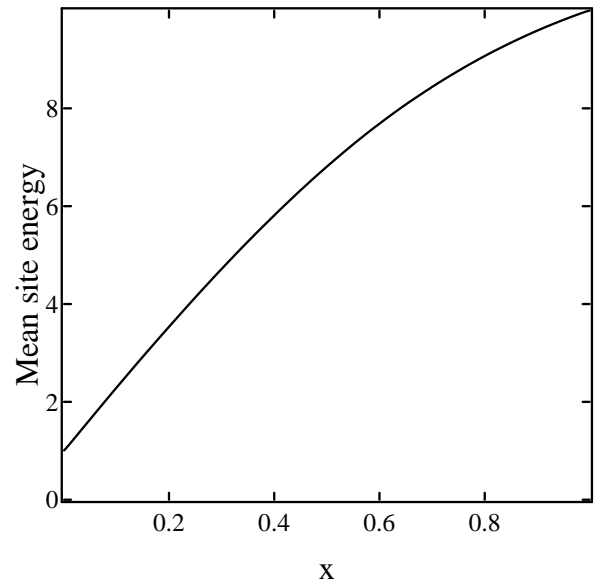

(a) Site energies, $N=1600$

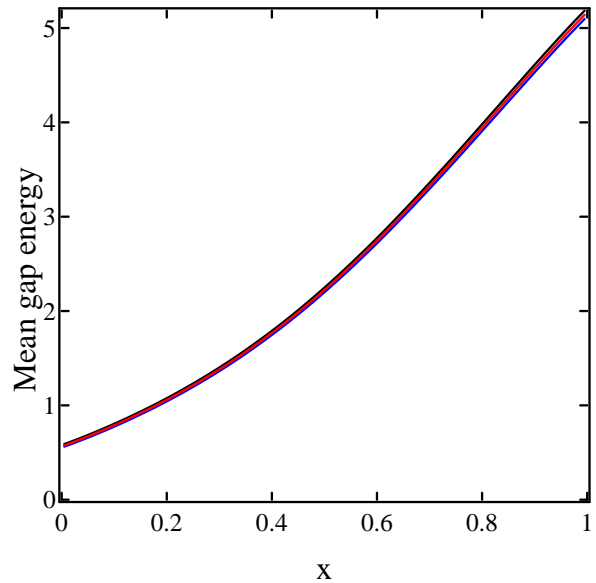

(b) Gap energies, $N=200$

Figure 1: Mean energy profiles for the exponential baths model. The bath temperatures are $T_{L}=1$ and $T_{R}=10$. Panel (a) shows the mean site energies $f_{N}$ for $N=1600$. In (b), we have superimposed the gap energy profiles $f_{N}^{+}, f_{N}^{-}$, and $f_{N}^{\sharp}$ for $N=200$. Numerical details: We impose a constraint that injected energies are $\geq 0.01$ but do not impose a high energy cutoff. We simulate the system for $10^{k}$ events (an "event" being one collision anywhere in the chain), increasing $k$ until the computed profile stabilizes. This occurred after $10^{13}$ events in (a), $4 \times 10^{11}$ events in (b).

\subsection{Local equilibrium properties}

This subsection is about local properties of NESS, by which we refer to marginals of the form $\hat{\mu}_{x, \ell, N}$ where $x \in(0,1), N$ is the length of the chain, $\ell \ll N$, and $\hat{\mu}_{x, \ell, N}$ is the marginal distribution of the NESS on the $\ell$-chain centered at site $[x N]$. For fixed (unequal) bath distributions, since the energy gradient on the $\ell$-chain tends to zero as $N \rightarrow \infty$, one might expect the $\hat{\mu}_{x, \ell, N}$ to resemble equilibrium distributions, i.e., invariant measures on chains with equal bath injections.

Theorem 2 gives an explicit formula for the equilibrium distribution $\mu_{\rho, N}$ of the $N$-chain when the bath distributions are $L=R=\rho$. This result is valid for very general $\rho$. Notice that when specialized to the case $\rho(\xi)=\beta e^{-\beta \xi}$, the density of $\mu_{\rho, N}$ has the familiar form $\frac{1}{Z} e^{-\beta H}$. More precisely, let us use $\left(x_{i}, v_{i}\right), v_{i}=\sigma_{i} \sqrt{\eta_{i}}$, as coordinates in the gaps instead of $\left(\eta_{i}, x_{i}, \sigma_{i}\right)$. Then

$$
d \mu_{\rho, N}=\left(\beta^{N} e^{-\beta \sum_{i} \xi_{i}} \Pi_{i} d \xi_{i}\right) \times\left(Z_{\beta}^{-(N+1)} e^{-\beta \sum_{i} v_{i}^{2}} \Pi_{i} d x_{i} d v_{i}\right)
$$

Returning to the situation of unequal exponential baths, one way to define local thermodynamic equilibrium (LTE) is to require that for every $x \in(0,1)$ and $\ell \in \mathbb{Z}^{+}$, as $N \rightarrow \infty$, the marginals $\hat{\mu}_{x, \ell, N}$ tend to a probability measure having the form in (1) with $N$ replaced by $\ell$ and $\beta=\beta(x)$ for some $\beta(x)>0$. For a more physical notion of LTE, one sometimes considers only $\ell$-chains for $1 \ll \ell \ll N$. 


\section{A. Single-site and single-gap marginals}

We consider marginal distributions of the NESS at single sites and single gaps at $[x N]$ where $x \in(0,1)$ is fixed and $N$ is varied. To show that the system does not tend to LTE (by any definition), it suffices to show that these marginals are not Gibbsian. This is because if marginals on $\ell$-chains tended to $\hat{\mu}_{x, \ell, N}$, projecting onto the single site or gap at $[x N]$ one would again obtain the a distribution of the form in (1).

Fig. 2 shows two examples of single-site marginal densities at $x=0.25$ and 0.6 for a chain of length $N=1600$. The plots are log-linear, so that exponential functions would appear as straight lines. Without a doubt both of the distributions shown are very far from exponential.

These distributions are compared to mixtures of the bath distributions, meaning distributions of the form

$$
a L(\xi)+(1-a) R(\xi), \quad a=a(x) \in(0,1)
$$

In Fig. 2, open squares represent empirical data (from simulations), while the solid curves are given by the formulas for mixtures with $a$ chosen in each of the two cases to fit the data best. Given the numerical cutoffs, etc., we think the fits between empirical data and the mixtures curves are excellent. Plots (not shown) at other locations evenly spaced along the chain show the same phenomenon, with the "knee" moving steadily to the left as $x$ increases.

Likewise, single-gap marginals are found to be mixtures: At gaps adjacent to $[x N]$ for fixed $x$, instead of $Z_{\beta}^{-1} e^{-\beta v^{2}}$ for some $\beta=\beta(x)$, we find numerically the marginals to be of the form

$$
a \cdot \frac{e^{-\beta_{L} v^{2}}}{Z_{\beta_{L}}}+(1-a) \cdot \frac{e^{-\beta_{R} v^{2}}}{Z_{\beta_{R}}}
$$

for some $a=a(x)$, in clear violation of the prescription of Gibbs for Hamiltonian systems in equilibrium.

We explain how we arrived at the idea of "mixtures": First, the energies in a chain should reflect those injected, and if there is a nontrivial discrepancy between $\beta_{L}$ and $\beta_{R}$, it is difficult to imagine having an abundance of the energies in the "middle" to constitute all the exponential distributions along the chain. We are also influenced by the following stochastic model, which can be thought of as a "zerothorder" approximation to our Hamiltonian systems.

\section{The random swaps model}

This is a stochastic model defined by $N$ random variables $\xi_{1}, \cdots, \xi_{N}$, to be thought of as energies, located at sites $1, \cdots, N$. As usual there are two baths, situated at sites 0 and $N+1$. At bonds between sites $i$ and $i+1$ for $i \in\{0,1, \cdots, N\}$ are exponential clocks which ring independently at rate 1 . When a clock goes off, energies between the 2 sites are swapped. That is to say, for $i \neq 0, N$, when the clock between sites $i$ and $i+1$ rings, the values of $\xi_{i}$ and $\xi_{i+1}$ are interchanged. Swapping energy with 


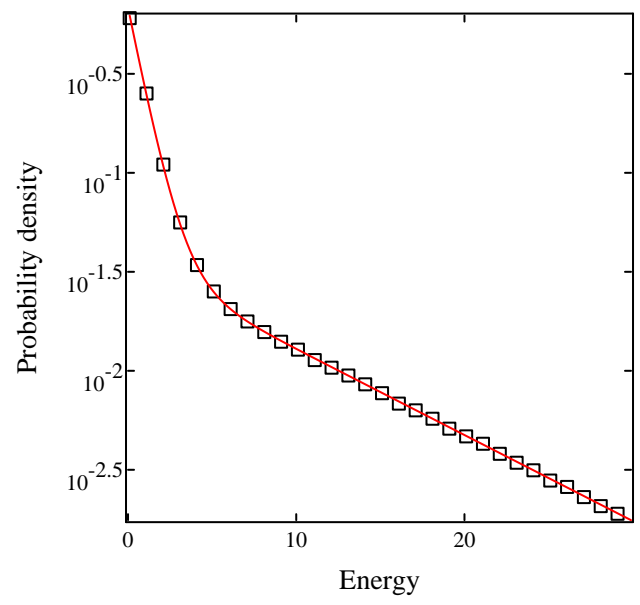

(a) $x=0.25$

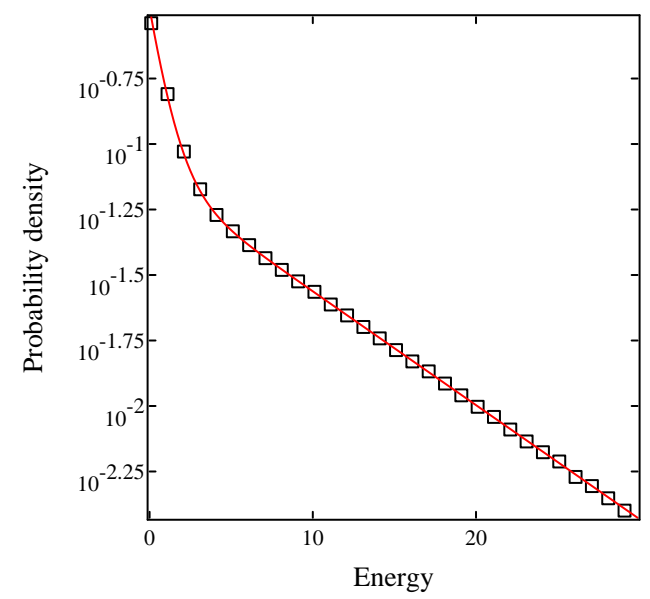

(b) $x=0.6$

Figure 2: Site energy distributions for the exponential baths model in log-linear scales. The parameters are $T_{L}=1, T_{R}=10, N=1600$. Empirical data are plotted in open squares; solid curve represents the mixture of left and right bath distributions that best fits the data (in the total variation norm).

the left bath means that $\xi_{1}$ is replaced by an energy drawn randomly from the left bath distribution $L(\xi)$, and similarly for $\xi_{N}$ and the right bath, which has distribution $R(\xi)$. The energies emitted by each bath are assumed to be i.i.d. and independent of emissions by the other bath. A model along similar lines was studied in [10].

Proposition 6.1 In the random swaps model, for each $i \in\{1, \cdots, N\}$, the marginal distribution $\rho_{i}$ of the unique NESS is given by

$$
\rho_{i}=\left(\frac{N+1-i}{N+1}\right) L+\left(\frac{i}{N+1}\right) R
$$

for arbitrary bath distributions $L$ and $R$.

Sketch of proof: First, we distinguish only between whether an energy is "from the left" or "from the right". In this reduced system, it is easy to see that there is a unique steady state, the single-site marginals of which are, by definition, mixtures of these two kinds of energies. Since the trajectory of each energy, from the time it enters the system to when it leaves, is that of a simple unbiased random walk, the weights in the formula for $\rho_{i}$ follow from basic recursive relations. Finally, each of the energies in this reduced system can be assigned independently any one of the allowed values, so that the marginals are really mixtures of $L$ and $R$.

Since Proposition 6.1 holds for arbitrary bath distributions $L$ and $R$, it is natural to ask if a similar result holds for the Hamiltonian chain with non-exponential baths. We investigated this question and found the answer to be negative. For example, when 


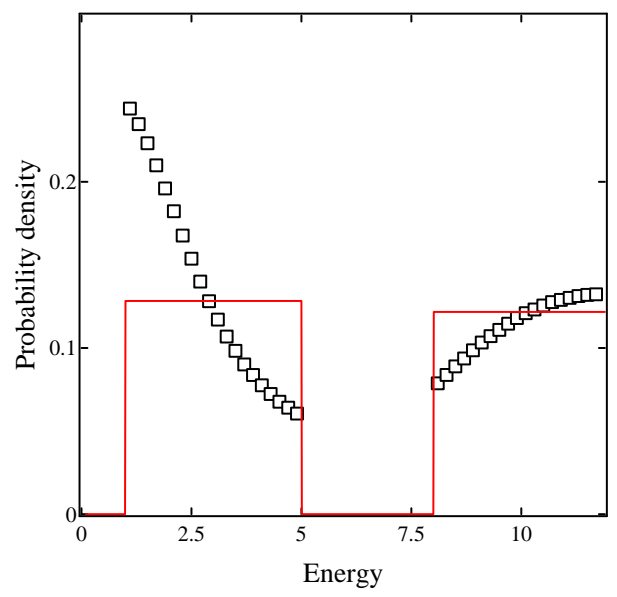

Figure 3: An example to show that site marginals are not necessarily mixtures. Bath distributions here are uniform distributions: $L(\xi)$ is supported on $[1,5], R(\xi)$ on $[8,12]$; $N=2000$. Empirical data are plotted in open squares as above.

$L$ and $R$ are uniform distributions, marginal distributions are far from mixtures of uniform distributions; see Fig. 3. When the densities of $L$ and $R$ have shapes closer to exponential distributions, single-site marginals are closer to mixtures, but noticeable differences were seen in each of the half-dozen or so cases tested.

The numerical results above raise the following questions:

(A) In the case of exponential baths, are single-site marginals as $N \rightarrow \infty$ genuinely mixtures of the two bath distributions, or are they simply close to mixtures?

(B) If the mixtures result here is exact, are exponential baths the only distributions that have this property, and if so, what are the underlying reasons?

Independently of the answer to (A), we believe we have shown very definitively that local marginal distributions do not have the form in (1). Hence the concept of LTE does not apply to this class of Hamiltonian chains.

\section{B. Vanishing of spatial correlations}

We investigate next if, as $N \rightarrow \infty, \hat{\mu}_{x, \ell, N} \rightarrow \mu_{\rho, \ell}$ where $\rho$ is the mixture found in Paragraph A. Since spatial correlations are expected to be largest between adjacent sites and gaps, we verify only that

(i) marginal distributions on two adjacent sites are product measures;

(ii) marginal distributions on two adjacent gaps are product measures, and the directions of travel are independent;

(iii) marginal distributions on a site and its adjacent gap are product measures, with gap density $=$ const $\cdot \rho(\eta) / \sqrt{\eta}$ where site density $=\rho$. 


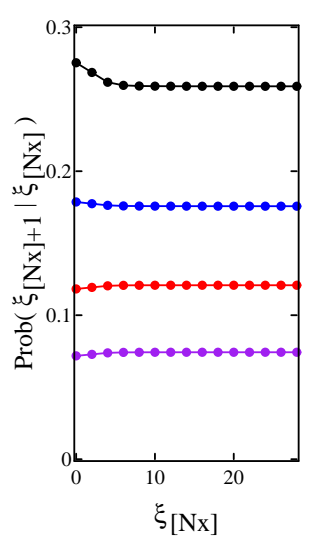

(a) $N=100$

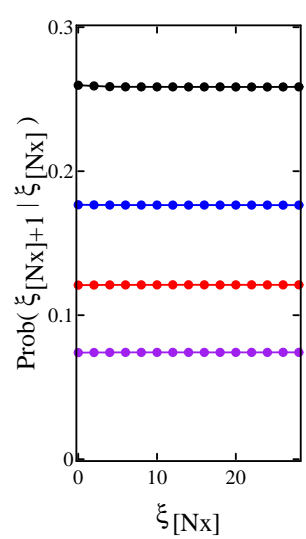

(b) $N=1600$

Figure 4: Conditional probabilities in adjacent sites for the exponential-baths model. The 4 curves in each panel show $P\left(\xi_{[N x]+1} \in I \mid \xi_{[N x]} \in[2 k, 2(k+1)]\right)$ for $k=0,1,2, \cdots, 14$ and $I=[0,2],[2,4],[4,6]$, and $[6,8]$ (top to bottom). In both panels, $x=0.6$. Bath parameters are $T_{L}=1, T_{R}=10$.

A sample of simulation results in support of (i) is shown in Fig. 4. In the horizontal axis are values of $\xi_{i}$ for $i=[0.6 N]$, and plotted are conditional distributions of $\xi_{i+1}$ given the various values of $\xi_{i}$. Here we group the values of $\xi_{i+1}$ into intervals $(0,2),(2,4),(4,6)$, and $(6,8)$, the 4 graphs representing the conditional probabilities of $\xi_{i+1}$ being in each one of these intervals given the value of $\xi_{i}$ in the horizontal axis. The two plots, for $N=100$ and 1600 respectively, show weak dependence of $\xi_{i}$ and $\xi_{i+1}$ in the first and close to zero dependence for the longer chain.

A small sample of data is shown in Table 5 to illustrate the combinations of joint densities that need to be checked to verify (ii). Here we focus on two adjacent gaps at $x=0.6$, and distinguish only between "high" and "low" energies, referring to energies $\eta>3$ as "high" and $\eta<3$ as "low". The fractions of time in all configurations of highlow and $\left(\sigma_{i}, \sigma_{i+1}\right)$, referring to the directions of travel in these gaps, are tabulated.

Finally, we have computed energy distributions for adjacent sites and gaps at various sites along the chain. The data confirm property (iii) above.

Remark. The "local product structure" finding above may be valid beyond the case of exponential baths. Our simulations show that it holds both for the uniform distribution models in Fig. 3 and for the "two-energy models" discussed in the next section. Since the latter are rather "extreme" from many points of view, it gives reason to conjecture that this phenomenon - which is very natural - may hold widely. 


\begin{tabular}{|c|c|c|c|c|c|}
\hline \multicolumn{6}{|c|}{$P\left(\eta_{i+1}<3 \mid \eta_{i}<3\right)=0.775=P\left(\eta_{i+1}<3 \mid \eta_{i}>3\right)$} \\
\hline \multicolumn{3}{|c|}{$\eta_{i}>3, \eta_{i+1}>3$} & \multicolumn{3}{|c|}{$\eta_{i}>3, \eta_{i+1}<3$} \\
\hline & $\sigma_{i}=+$ & $\sigma_{i}=-$ & & $\sigma_{i}=+$ & $\sigma_{i}=-$ \\
\hline$\sigma_{i+1}=+$ & 0.013 & 0.013 & $\sigma_{i+1}=+$ & 0.043 & 0.044 \\
\hline$\sigma_{i+1}=-$ & 0.013 & 0.013 & $\sigma_{i+1}=-$ & 0.043 & 0.043 \\
\hline \multicolumn{3}{|c|}{$\eta_{i}<3, \eta_{i+1}>3$} & \multicolumn{3}{|c|}{$\eta_{i}<3, \eta_{i+1}<3$} \\
\hline & $\sigma_{i}=+$ & $\sigma_{i}=-$ & & $\sigma_{i}=+$ & $\sigma_{i}=-$ \\
\hline$\sigma_{i+1}=+$ & 0.044 & 0.043 & $\sigma_{i+1}=+$ & 0.151 & 0.150 \\
\hline$\sigma_{i+1}=-$ & 0.044 & 0.043 & $\sigma_{i+1}=-$ & 0.150 & 0.150 \\
\hline
\end{tabular}

Table 5: Joint distributions of gap energies conditioned on directions of travel. Here, $N=1600$ and $i=[0.6 \mathrm{~N}]$. The numbers are raw probabilities of the specified events.

\section{Two-energy Models}

The bath distributions in this section are sharply peaked Gaussians truncated at 0 . In the simulations shown, these Gaussians have means 1 and 5, and their standard deviations are chosen to be 0.2 , so that the probability of being within \pm 0.5 of the means is roughly 99\%. Even though these systems are small perturbations of integrable models, we know from Theorem 1 that all of their invariant measures are ergodic.

\subsection{Macroscopic energy profiles and local distributions}

Fig. 6(a) shows site-energy profiles $f_{N}$ for a range of $N$. Unlike the exponential case, these profiles vary substantially with $N$ : For $N=10$ (not shown), the profile is essentially flat; as $N$ increases, it acquires a gradient and appears to be stabilizing, but even at $N=3200$, the profile is still moving a little. From these profiles, one suspects - correctly - that for (very) small $N$, most energies move monotonically along the chain, entering from one end and exiting at the other. As $N$ increases, some of the energies turn around, some doing so a number of times before exiting, creating a gradient in the profile.

Fig. 6(b) shows profiles for gap energies with specified directions, showing that for the chain with $N=200, f_{N}^{+}$and $f_{N}^{-}$are substantially different, with more energy 1s moving to the right and $5 \mathrm{~s}$ moving to the left. This is to be contrasted with Fig. 1(b), which shows that for exponential baths, this left-right discrepancy has by and large vanished by $N=200$. Indeed in the present model, $f_{N}^{+}$and $f_{N}^{-}$remain quite far apart in some of the gaps even at $N=3200$, leaving open the question whether or not the distributions conditioned on $\sigma_{i}=+$ and - will eventually equalize. A follow-up investigation is discussed in Sect. 7.2.

With regard to local distributions, computations on site-to-site, gap-to-gap, and site-to-gap correlations similar to those in Section 6 were carried out. Independence 


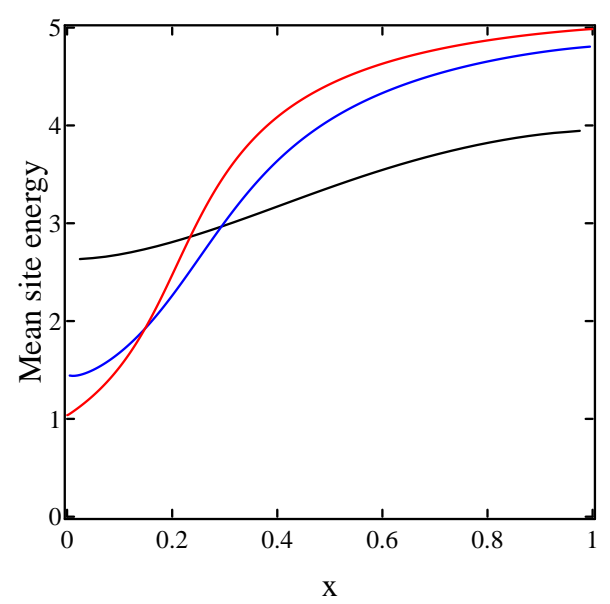

(a) Mean site energies

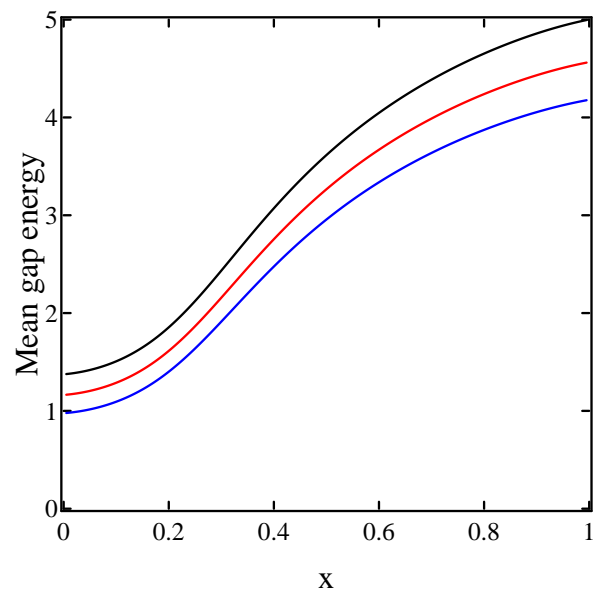

(b) Mean gap energies

Figure 6: Mean energy profiles for two-energy model. Panel (a) shows mean site energies; the curves are $N=40,200$, and 3200 , in order of increasing values at $x=1$. Panel (b) shows mean gap energies $f_{N}^{-}, f_{N}^{\sharp}$, and $f_{N}^{+}$(top to bottom) for $N=200$. The rules used to determine when a steady state is reached are as Fig. 1. See text for the bath distributions.

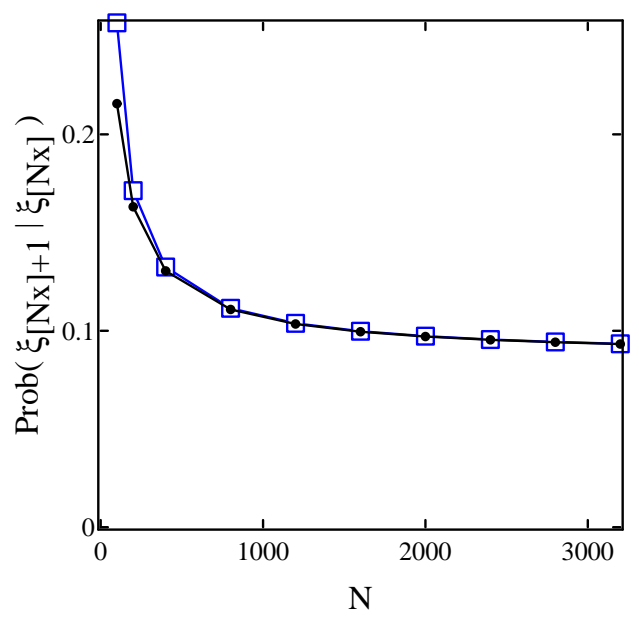

Figure 7: Nearest-neighbor conditional probabilities in the two-energy model. This plot shows $P\left(\xi_{[N x]+1}<3 \mid \xi_{[N x]}<3\right)$ (open squares) and $P\left(\xi_{[N x]+1}<3 \mid \xi_{[N x]}>3\right)$ (solid discs) as functions of $N$. The location is $x=0.6 ; N$ ranges from 100 to 3200 . 
was found to be achieved already at relatively small $N$.

Fig. 7 captures the two main points: The two curves representing $P\left(\xi_{[0.6 N]+1}=\right.$ $\left.1 \mid \xi_{[0.6 N]}=k\right), k=1$ and 5 , are virtually on top of each other for $N \geq 400$, illustrating the rapid vanishing of correlations between adjacent sites, while both curves continue to have a slightly negative slope at $N=3200$, illustrating the slow convergence of mean site energies.

\subsection{Ballistic transport vs. diffusion: a phenomenological ex- planation}

The purpose of this subsection is to examine more closely the way in which energy is transported from one end of the chain to the other under near-integrable dynamics. Since the vast majority of the energies in this system are very close to 1 or 5 , let us assume there are only two kinds of energies in the system. For definiteness, we adopt the view in Sect. 1.3, and focus on the movement of $1 \mathrm{~s}$ on the right side of the chain.

We will demonstrate that modulo certain time changes, the statistics generated by the movements of these energies resemble those of a particle undergoing a 1-D diffusion with a spatially-varying diffusion coefficient. We begin by introducing an especially simple model which will be used for comparison purposes:

Model A. Consider a Markov chain with state space $\{0,1, \cdots, N+1\}$ defined as follows: Starting from 1, one performs a simple, unbiased random walk until either 0 or $N+1$ is reached, then returns to 1 to start over again. For $1 \leq i \leq N-1$, let $\ell_{n}(i)$ and $r_{n}(i)$ denote the number of left and right crossings respectively between sites $i$ and $i+1$ in the first $n$ steps, and let $\mathbb{E}_{N, i}=\lim _{n \rightarrow \infty} \mathbb{E}_{N}\left[\ell_{n}(i)\right] / \mathbb{E}_{N}\left[r_{n}(i)\right]$. Here are some easy facts:

(i) For all $N$ and $i, 0<\mathbb{E}_{N, i}<1$.

(ii) For each $N, \mathbb{E}_{N, i}$ decreases as $i$ increases.

(iii) For each $x \in(0,1), \mathbb{E}_{N,[x N]}$ increases to 1 as $N \rightarrow \infty$.

These facts follow from the following observations: Fix $i$ and $N$, and focus only on left and right crossings between sites $i$ and $i+1$. Then a left crossing is necessarily followed by a right crossing, while a right crossing can be followed by either. The probability that a right crossing is followed by another right crossing is equal to the probability of starting from site $i+1$ and reaching site $N+1$ before site $i$ in the random walk. The numbers $\mathbb{E}_{N, i}$ can be explicitly estimated if one so desires.

To bring this simple model closer to our Hamiltonian chains, we first identify some relevant features of the chains. The following idea is used in the rigorous part of this paper (Lemmas 4.2 and 4.4): Consider a scenario in which an energy 1 is in the midst of many energy $5 \mathrm{~s}$, i.e. in some segment of the chain, $\xi_{j}=\eta_{j}=5$ for all $j$ except for a single $\hat{j}$ where $\eta_{\hat{j}}=1$. Then the energy 1 will move monotonically in some direction until the pattern is disrupted by the approach of another (oncoming) energy 1. After such a "collision" a variety of things can happen; the resulting motion of the energies 
depends on the details of the interaction. Since the energy profiles have nonzero gradient (see Fig. 6), 1s are more sparse on the right side of the chain. Thus the closer to the right end, the larger the "mean free distance" between "collisions" of energy 1s.

With regard to the ratio of left to right crossings (and not the actual number of crossings per unit time), the observations above suggest the following modification of Model A:

Model B. Let $\lambda:(0,1) \rightarrow(0, \infty)$ be a function that monotonically increases from 1 to $\infty$ on some subinterval $\left(x_{*}, 1\right) \subset(0,1)$. We consider a process similar to that in Model A but with transition rules modified as follows: when at site $i=[x N]$, go $X$ sites to the left/right with probability $\frac{1}{2} / \frac{1}{2}$ where $X$ is an exponential random variable with mean $\lambda(x)$, and return as before to 1 to start over once 0 or $N+1$ is reached.

First we confirm numerically that in terms of the statistics generated, Model B gives a good approximation of movements of energy 1s on the right side of the real chain. (Obviously, we do not claim that the two models are equivalent.) We study the lengths of runs of randomly picked $1 \mathrm{~s}$ in specified segments of the chain, a run being defined to be a consecutive sequence of moves in the same direction. Some mean length-of-runs are tabulated in Fig. 8(a). The numbers in each row increase as one moves to the right, a trend consistent with the environment becoming increasingly dominated by energy 5s. As $N$ increases, these means stabilize, as one would expect them to when the local marginals tend to a distribution of the form $\mu_{\rho, \ell}$. Histograms of lengths of runs within specified intervals are plotted and found to have roughly exponential distributions for large $N$; one such plot is shown in Fig. 8(b).

Returning to Models A and B, observe that they are qualitatively similar if we imagine that the sites in Model B are "packed closer and closer" as $x \rightarrow 1$. More precisely, the ratio of left to right crossings at site $[x N]$ in Model B should resemble that at $[y N]$ in Model A for some $y>x$, with $(1-y) /(1-x) \rightarrow 0$ as $x \rightarrow 1$. Properties (i-iii) in Model A therefore pass to Model B. Assuming they pass from there to our Hamiltonian chain, one would conclude that for our chain:

- left-right traffic will equalize everywhere as $N \rightarrow \infty$;

- this equalization occurs more slowly than at corresponding sites in Model A, the discrepancy increasing as $x \rightarrow 1$.

Simulations to compute the ratios of left/right crossings were performed. The results, shown in Fig. 8(c), are very much consistent with the predictions above: for each $N$, the $x \mapsto \operatorname{Prob}\left(\sigma_{[N x]}=-\right) / \operatorname{Prob}\left(\sigma_{[N x]}=+\right)$ curves are decreasing, and at each $x \in(0,1)$, this ratio increases with $N$ and appears to head toward 1 .

Remarks on connection with theory. We have used the fact, proven rigorously in Sect. 4.3, that in regions of the chain occupied by significantly more energies of one 


\begin{tabular}{c|ccccccc} 
& \multicolumn{7}{|c}{ Interval of $x$} \\
& {$[0.2,0.4]$} & {$[0.3,0.5]$} & {$[0.4,0.6]$} & {$[0.5,0.7]$} & {$[0.6,0.8]$} & {$[0.7,0.9]$} & {$[0.8,1.0]$} \\
\hline$N=400$ & 5.7 & 5.9 & 6.6 & 8.1 & $10^{*}$ & - & - \\
$N=1600$ & 5.6 & 6.0 & 7.4 & 9.6 & 13 & 20 & $35^{*}$ \\
$N=3200$ & 5.5 & 6.1 & 7.5 & 10 & 14 & 21 & 40
\end{tabular}

(a) Mean lengths of runs

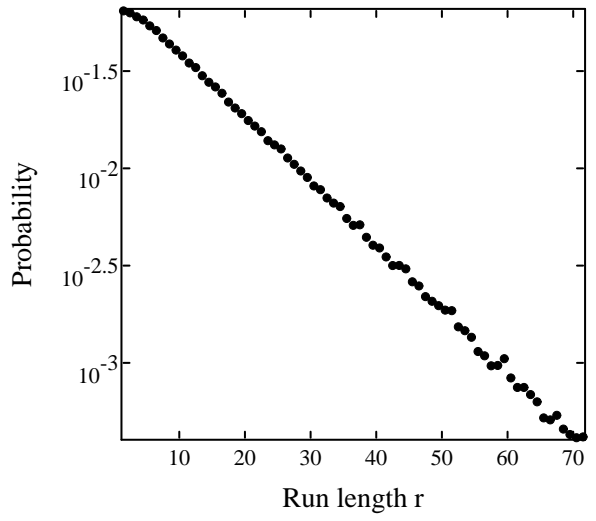

(b) Prob(run length $=r$ )

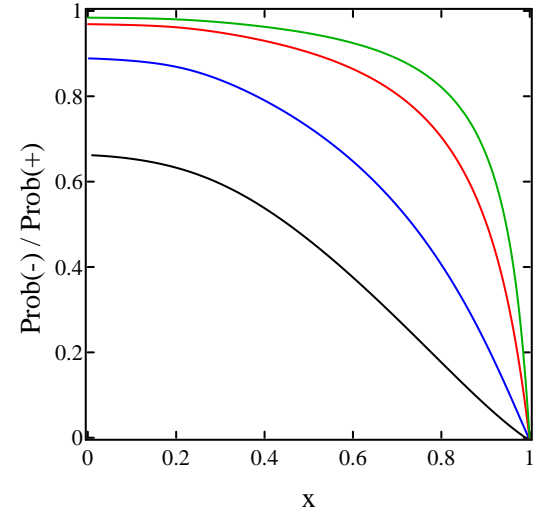

(c) $\operatorname{Prob}\left(\sigma_{[x N]}=-\right) / \operatorname{Prob}\left(\sigma_{[x N]}=+\right)$

Figure 8: Statistics on lengths of runs. (a) Mean lengths of runs in different intervals $[a, b]$. Note that runs that reach the boundary of $[a, b]$ are artificially cut short by our numerical procedure (see "numerical details" below); we do not consider averages based on too many $(>10 \%)$ such runs meaningful and have omitted those averages. $(*)$ marks averages for which $5-10 \%$ of the runs were cut short. (b) Log-linear plot of the histogram of run-lengths in the interval $[0.6,0.8]$, for $N=3200$. (c) The ratio $P\left(\eta_{i} \approx 1, \sigma=-\right) / P\left(\eta_{i} \approx 1, \sigma=+\right)$ as a function of $x=i / N$. The curves are, from bottom to top, $N=100,400,1600,3200$. Numerical details for (a) and (b): For a given interval $[a, b]$, we begin with a suitably prepared system and wait until an energy $\approx 1$ hits the site at $x=(a+b) / 2$. The energy is tracked until it hits either $x=a$ or $x=b$; the lengths of runs executed by this energy are recorded. This process is repeated (after a delay to ensure that the energies are not too correlated). Each data point in Table (a) based on $10^{4}-10^{6}$ runs collected this way. 
kind than the other, the ones with low density will, on average, have relatively long runs. The same is true, in fact, for the energies with high density. Our rigorous work leaves untreated the situation where the two energies occur in roughly comparable proportions. For these regions, we have seen via simulations that the mean lengths of runs tend still to be greater than 2, the value for simple unbiased random walks. For example, when $T_{L}=1$ and $T_{R}=5$, the region with the most even mix of $1 \mathrm{~s}$ and $5 \mathrm{~s}$ is $[0.2,0.4]$, and there the mean run-length for $1 \mathrm{~s}$ is $>5$ (see Fig. 8(a)). Thus the phenomena discussed in this subsection appear to be valid for both energies on a good part of the chain.

\section{Summary and Conclusion}

We considered a Hamiltonian chain with many conserved quantities and studied its nonequilibrium steady states when the two ends of the chain are put in contact with unequal heat baths. Our main findings are:

Rigorous results. First, under mild restrictions on the bath distributions $L(\xi)$ and $R(\xi)$, we proved ergodicity of the invariant measure (assuming existence). Second, we identified a class of equilibrium measures $\left\{\mu_{\rho, N}\right\}$ where $\mu_{\rho, N}$ is the unique invariant probability on the $N$-chain with $L=R=\rho$; the measures $\mu_{\rho, N}$ are product measures.

Numerical results. Simulations were performed for chains with two kinds of bath distributions: exponential distributions and sharply peaked Gaussians, the latter giving rise to what is called the "two-energy model".

1. We demonstrated numerically that (a) NESS exist for finite $N$ and mean energy profiles converge as $N \rightarrow \infty$; (b) as $N \rightarrow \infty$ local marginals at $x \in(0,1)$ tend to measures of the form $\mu_{\rho, \ell}$ for some distribution $\rho=\rho(x)$.

2. For exponential bath distributions, the limits of local marginals are definitively not Gibbs measures, i.e., this chain violates the concept of LTE. The marginals appear to be weighted averages of Gibbs measures.

3. For the 2-energy model, the paths traced out by energies resemble the sample paths of a random walk with a bias in favor of continuing in the same direction, with this bias increasing as $x \rightarrow 0$ or 1 . We conclude that the resulting transport behavior is more normal, i.e., more diffusive, than one might have expected given the integrable character of the dynamics.

\section{References}

[1] C. Bernardin, S. Olla, "Fourier's law for a microscopic model of heat conduction," J. Stat. Phys. 118 (2005) pp. 271-289 
[2] L. Bertini, A. De Sole, D. Gabrielli, G. Jona-Lasinio, C. Landim, "Macroscopic fluctuation theory for stationary non-equilibrium states," J. Statist. Phys. 107 (2002) pp. 635-675

[3] F. Bonetto, J. Lebowitz, and L. Rey-Bellet, "Fourier law: a challenge to theorists," in Mathematical Physics 2000, edited by A. Fokas, A. Grigoryan, T. Kibble, and B. Zegarlinski, Imp. Coll. Press, London (2000)

[4] J. Bricmont and A. Kupiainen, "Fourier's law from closure equations," Phys. Rev. Lett. 98 (2007) 214301

[5] J. Bricmont and A. Kupiainen, "Towards a derivation of Fourier's law for coupled anharmonic oscillators," Comm. Math. Phys. 274 (2007) 555-626

[6] P. Collet and J.-P. Eckmann, "A model of heat conduction," preprint (2008)

[7] P. Collet, J.-P. Eckmann, and C. Mejía-Monasterio, "Superdiffusive heat transport in a class of deterministic one-dimensional many-particle Lorentz gases," preprint (2008)

[8] B. Derrida, "Non-equilibrium steady states: fluctuations and large deviations of the density and of the current," J. Stat. Mech. (2007) P07023

[9] B. Derrida, J. L. Lebowitz, E. R. Speer, "Large deviation of the density profile in the steady state of the open symmetric simple exclusion process," J. Statist. Phys. 107 (2002) pp. 599-634

[10] A. Dhar and D. Dhar, "Absence of local thermal equilibrium in two models of heat conduction," Phys. Rev. Lett. 82 (1999) 480-483

[11] S. R. de Groot, P. Mazur, Non-equilibrium Thermodynamics, North-Holland (1962)

[12] J.-P. Eckmann and M. Hairer, "Non-equilibrium statistical mechanics of strongly anharmonic chains of oscillators," Commun. Math. Phys. 212 (2000) pp. 105-164

[13] J.-P. Eckmann and P. Jacquet, "Controllability for chains of dynamical scatterers," Nonlinearity 20 (2007) 1601-1617

[14] J.-P. Eckmann, C. Mejía-Monasterio, and E. Zabey, "Memory effects in nonequilibrium transport for deterministic Hamiltonian systems," to appear in J. Stat. Phys. (2006)

[15] J.-P. Eckmann, C.-A. Pillet, L. Rey-Bellet, "Non-equilibrium statistical mechanics of anharmonic chains coupled to two heat baths at different temperatures," Comm. Math. Phys. 201 (1999) 657-697 
[16] J.-P. Eckmann and L.-S. Young, "Nonequilibrium energy profiles for a class of 1-D models," Comm. Math. Phys. 262 (2006) pp. 237-267

[17] P. Gaspard and T. Gilbert, "Heat conduction and Fourier's law in a class of many particle dispersing billiards," New J. Phys. 10 (2008) 103004

[18] C. Kipnis, C. Landim, Scaling Limits of Interacting Particle Systems, Berlin: Springer-Verlag (1999)

[19] C. Kipnis, C. Marchioro, E. Presutti, "Heat flow in an exactly solvable model," J. Stat. Phys. 27 (1982) pp. 65-74

[20] H. Larralde, F. Leyvraz, C. Mejía-Monasterio, "Transport properties of a modified Lorentz gas," J. Stat. Phys. 113 (2003) pp. 197-231

[21] S. Lepri, R. Livi, and A. Politi, "Thermal conduction in classical low-dimensional lattices," Physics Reports 377 (2003)

[22] B. Li, G. Casati, J. Wang, and T. Prosen, "Fourier Law in the alternate-mass hard-core potential chain," Phys. Rev. Lett. 92 (2004) 254301

[23] K. K. Lin and L.-S. Young, "Correlations in nonequilibrium steady states of random-halves models," J. Stat. Phys. 128 (2007) 607-639

[24] S. Olla, S. R. S. Varadhan, and H. T. Yau, "Hydrodynamical limit for a Hamiltonian system with weak noise," Comm. Math. Phys. 155 (1993) 523-560

[25] K. Rateitschak, R. Klages, and G. Nicolis, "Thermostating by deterministic scattering: the periodic Lorentz gas," J. Stat. Phys. 99 (2000) 1339-1364

[26] K. Ravishankar, L.-S. Young, "Local thermodynamic equilibrium for some stochastic models of Hamiltonian origin," J Stat. Phys. 128 (2007) 641-665

[27] L. Rey-Bellet and L. E. Thomas, "Exponential convergence to non-equilibrium stationary states in classical statistical mechanics," Comm. Math. Phys. 225 (2002) 305-329

[28] Z. Rieder, J. L. Lebowitz, and E. Lieb, "Properties of a harmonic crystal in a stationary nonequilibrium state," J. Math. Phys. 8 (1967)

[29] H. Spohn, "Long range correlations for stochastic lattice gases in a nonequilibrium steady state," J. Phys. A 16 (1983) pp. 4275-4291 